\title{
Compact formulas for the completed mock modular forms
}

\section{Tohru Eguchi $^{a}$ and Yuji Sugawara ${ }^{b}$}

${ }^{a}$ Department of Physics and Research Center for Mathematical Physics, Rikkyo University, Tokyo 171-8501, Japan

${ }^{b}$ Department of Physical Sciences, College of Science and Engineering, Ritsumeikan University, Shiga 525-8577, Japan

E-mail: tohru.eguchi@gmail.com, ysugawa@se.ritsumei.ac.jp

ABSTRACT: In this paper we present a new compact expression of the elliptic genus of $\mathrm{SL}(2) / \mathrm{U}(1)$-supercoset theory by making use of the 'spectral flow method' of the pathintegral evaluation. This new expression is written in a form like a Poincaré series with a non-holomorphic Gaussian damping factor, and manifestly shows the modular and spectral flow properties of a real analytic Jacobi form. As a related problem, we present similar compact formulas for the modular completions of various mock modular forms which appear in the representation theory of $\mathcal{N}=2,4$ superconformal algebras.

We further discuss the generalization to the cases of arbitrary spin-structures, that is, the world-sheet fermions with twisted boundary conditions parameterized by a continuous parameter. This parameter is naturally identified with the ' $u$-variable' in the Appell-Lerch sum.

KEYwords: Conformal Field Models in String Theory, Superstring Vacua, Black Holes in String Theory

ARXIV EPRINT: 1407.7721 


\section{Contents}

1 Introduction and summary 1

2 Revisiting elliptic genus of $\mathrm{SL}(2) / \mathrm{U}(1)$-supercoset model as a non-holomorphic Poincaré series $\quad 3$

2.1 A refined calculation of elliptic genus based on the spectral flow method 3

$\begin{array}{lll}2.2 & \text { Relations between the new and old expressions of modular completions } & 7\end{array}$

$\begin{array}{lll}3 & \text { Extension to general spin structures } & 10\end{array}$

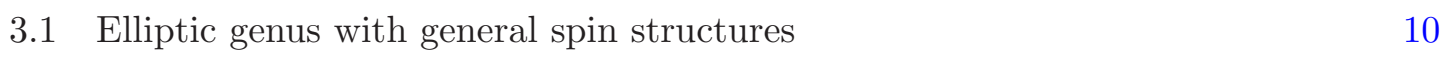

$\begin{array}{ll}3.2 \text { Modular completions of superconformal characters } & 15\end{array}$

$\begin{array}{lr}\text { A Notations and useful formulas } & 18\end{array}$

$\begin{array}{ll}\text { B Proof of (3.8) } & 21\end{array}$

$\begin{array}{ll}\text { C Proof of (3.15) } & 22\end{array}$

D Summary of modular completions with general spin structures $\quad 24$

\section{Introduction and summary}

It is known that superconformal field theories with non-compact target space possess interesting theoretical issues; for instance, in order to regulate its IR divergences we may sometimes have to sacrifice its holomorphy property. A good laboratory to study them is the $\mathrm{SL}(2) / \mathrm{U}(1)$-supercoset theory, which is interpreted as the supersymmetric extension of the model of 2-dimensional black hole [1-4]. One of the important progress in the study of this model has been the appearance of non-holomorphicity in the elliptic genus [5-7]. This originates from the existence of gapless continuous spectrum of non-BPS states causing the IR-divergence in the theory. Quite interestingly, these studies have revealed the physical origin of a mathematical construction of 'modular completions' of mock modular forms [8]. Namely, it turned out that elliptic genera of SL(2)/U(1)-theory $[5,6]$ are written in terms of the modular completion of the mock modular form [8];

$$
\widehat{f}_{u}^{(k)}(\tau, z):=f_{u}^{(k)}(\tau, z)-\frac{1}{2} \sum_{m \in \mathbb{Z}_{2 k}} R_{m, k}(\tau, u) \Theta_{m, k}(\tau, 2 z), \quad\left(k \in \mathbb{Z}_{>0}\right) .
$$

Here the mock modular form (Appell-Lerch sum) is defined as

$$
f_{u}^{(k)}(\tau, z):=\sum_{n \in \mathbb{Z}} \frac{q^{k n^{2}} y^{2 k n}}{1-y w^{-1} q^{n}}, \quad\left(q \equiv e^{2 \pi i \tau}, y \equiv e^{2 \pi i z}, w \equiv e^{2 \pi i u}\right)
$$


and has an anomalous modular transformation law (this function with $u=0$ is closely related to the character of massless representations of $\mathcal{N}=4$ superconformal algebra [9, 10]). Anomalous transformation law is compensated by the second term in the r.h.s. of (1.1) with

$$
\begin{array}{r}
R_{m, k}(\tau, u):=\sum_{\nu \in m+2 k \mathbb{Z}}\left[\operatorname{sgn}(\nu+0)-\operatorname{Erf}\left\{\sqrt{\frac{\pi \tau_{2}}{k}}\left(\nu+2 k \frac{u_{2}}{\tau_{2}}\right)\right\}\right] w^{-\nu} q^{-\frac{\nu^{2}}{4 k}} \\
\left(\tau_{2} \equiv \operatorname{Im} \tau, u_{2} \equiv \operatorname{Im} u\right)
\end{array}
$$

which has a non-holomorphic dependence on $\tau$. Completed mock modular form $\hat{f}_{u}^{(k)}(\tau, z)$ has a well-defined transformation law as a Jacobi form of weight 1 and index $k$. (See appendix A for the convention of error function $\operatorname{Erf}(x)$, theta function $\Theta_{m, k}(\tau, z)$ and Jacobi forms.) This means that the elliptic genus of $\mathrm{SL}(2) / \mathrm{U}(1)$-supercoset is described by a non-holomorphic generalization of a Jacobi form.

Moreover, the torus partition function has also been analyzed in [6], and is shown to be expanded in terms of the modular completions of extended discrete characters (see appendix D) as well as continuous (non-BPS) characters. More recently, in literatures [1113], the elliptic genera of the $\mathrm{SL}(2) / \mathrm{U}(1)$-theory as well as some generalized models have been analyzed using the formulation of the gauged linear sigma model as proposed in [14].

In this paper, we shall propose a method of deriving compact expressions for the completed mock modular forms that makes their modular properties manifest. To this end, we adopt the technique of spectral flow method, which was partly studied in [15], rather than the method of character decomposition in [6]. Using the new method, we can simplify relevant calculations.

What is important is that we are naturally led to an expression of elliptic genus or completed mock modular forms as a sum over a 2-dimensional lattice

$$
\mathcal{Z}(\tau, z) \sim \sum_{m, n \in \mathbb{Z}} g_{(m, n)}(\tau, z)\left(\equiv \sum_{\lambda \equiv m \tau+n \in \Lambda} g_{\lambda}(\tau, z), \quad(\Lambda \equiv \mathbb{Z} \tau+\mathbb{Z})\right),
$$

where $g_{(m, n)}(\tau, z)$ behaves covariantly under modular transformations;

$$
g_{(m, n)}(\tau+1, z)=g_{(m, m+n)}(\tau, z), \quad g_{(m, n)}\left(-\frac{1}{\tau}, \frac{z}{\tau}\right)=e^{i \pi \frac{\hat{c}}{\tau} z^{2}} g_{(n,-m)}(\tau, z) .
$$

( $\hat{c}$ is defined in (2.1)). Therefore, this expression manifestly exhibits modular invariance as in the case of Poincaré or Eisenstein series. The two integers $m, n$ are identified with spectral parameters along the spatial and temporal cycles of a torus.

Let us summarize the main results of this paper;

(i) The main formula we will derive is given in (2.21). This is written in the form of nonholomorphic Poincaré type series mentioned above, and will be a most tranparent expression of elliptic genus of the cigar model. We will reach this formula in two ways: one is a direct evaluation of path-integration with the help of spectral flow expansion. The other is based on the properties of the modular completions of extended or 
irreducible discrete characters given in the previous papers $[6,15]$. Combining these two approaches, we can rewrite the function $\widehat{f}_{u=0}^{(k)}(\tau, z)(1.1)$ as given in (2.29). We can also express the modular completions of discrete characters in the forms of double series mentioned above. These are given in (2.31) and (2.32).

(ii) We also discuss the generalization to the cases of arbitrary spin-structures, that is, the world-sheet fermions with twisted boundary conditions parameterized by a continuous parameter. This continuous parameter is naturally identified with the ' $u$-variable' in the Appell-Lerch sum. The complete formula of rewriting $\widehat{f}_{u}^{(k)}(\tau, z)$ in terms of the non-holomorphic Poincaré series is given in (3.15).

\section{Revisiting elliptic genus of $\mathrm{SL}(2) / \mathrm{U}(1)$-supercoset model as a non-holomorphic Poincaré series}

\subsection{A refined calculation of elliptic genus based on the spectral flow method}

We shall start with the torus partition function with the general twist angles $z, \bar{z} \in \mathbb{C}$ (in the $\widetilde{\mathrm{R}}$-sector) of the cigar $\mathrm{SL}(2) / \mathrm{U}(1)$-supercoset model, which has been evaluated in [6]. ${ }^{1}$ Throughout this paper we set the level of SL(2) super-WZW model to be a real positive number $k$ (the level of bosonic part is $k+2$ ), which means that the central charge of this superconformal system is

$$
\hat{c}\left(\equiv \frac{c}{3}\right)=1+\frac{2}{k} .
$$

Because of the IR-divergence of cigar geometry, we have to introduce a suitable regularization. Here we shall adopt a 'refined' scheme of regularization. Namely, we start with the following regularized partition function ${ }^{2}\left(\mu \equiv \mu_{1}+i \mu_{2}, z \equiv z_{1}+i z_{2}, \quad \mu_{1}, \mu_{2}, z_{1}, z_{2} \in \mathbb{R}\right)$

$$
\boldsymbol{Z}_{\mathrm{reg}}(\tau, z, \bar{z} ; \epsilon)=k e^{2 \pi \frac{\hat{c}}{\tau_{2}}|z|^{2}-2 \pi \frac{k+4}{k \tau_{2}} z_{2}^{2}} \int_{\mathbb{C}} \frac{d^{2} \mu}{\tau_{2}} \sigma(\tau, \mu, z, \bar{z} ; \epsilon)\left|\frac{\theta_{1}\left(\tau, \mu+\frac{k+2}{k} z\right)}{\theta_{1}\left(\tau, \mu+\frac{2}{k} z\right)}\right|^{2} e^{-4 \pi z_{2} \frac{\mu_{2}}{\tau_{2}}} e^{-\frac{\pi k}{\tau_{2}}|\mu|^{2}}
$$

where we introduced a regularization factor $\sigma(\tau, \mu, z, \bar{z} ; \epsilon)(\epsilon>0)$ defined as

$$
\sigma(\tau, \mu, z, \bar{z} ; \epsilon):=\prod_{m_{1}, m_{2} \in \mathbb{Z}}\left[1-e^{-\frac{1}{\epsilon \tau_{2}}\left\{\left(s_{1}+m_{1}\right) \tau+\left(s_{2}+m_{2}\right)+\frac{2 z}{k}\right\}\left\{\left(s_{1}+m_{1}\right) \bar{\tau}+\left(s_{2}+m_{2}\right)+\frac{2 \bar{z}}{k}\right\}}\right],
$$

with $\mu \equiv s_{1} \tau+s_{2}, s_{i} \in \mathbb{R}, i=1,2$. Note that all the singularities of the integrand located at $\mu+\frac{2}{k} z \in \mathbb{Z} \tau+\mathbb{Z}$ that originate from the $\theta_{1}$-factor are removed by inserting (2.3) and the $\mu$-integral converges as long as $\epsilon>0$. We will later regard $\sigma(\tau, \mu, z, \bar{z} ; \epsilon)$ as a holomorphic function with respect to complex variables $s_{1}, s_{2}$.

Let us very briefly sketch how the torus partition function (2.2) is derived. See [6] for the details.

\footnotetext{
${ }^{1}$ See also [16-18] for the earlier works in which closely related analyses are presented.

${ }^{2}$ We shall denote the regularized partition function as ' $\boldsymbol{Z}_{\mathrm{reg}}(\tau, z, \bar{z} ; \epsilon)$ ' rather than ' $\boldsymbol{Z}_{\mathrm{reg}}(\tau, z ; \epsilon)$ ' although $\bar{z}$ is just the complex conjugate of $z$ in $(2.2)$. This is because we will later treat $z$ and $\bar{z}$ as two independent complex variables to derive the elliptic genus, while $\bar{\tau}$ is always the complex conjugate of $\tau$ through this paper.
} 
- According to the standard analysis of gauged WZW models [19-21], the relevant calculation reduces to that of free fields, except for the integral of modulus $\mu$ that is coupled to the anomaly-free U(1)-current we should gauge;

$$
\mathcal{J} \equiv j^{3}+\psi^{+} \psi^{-}+i \sqrt{k} \partial Y
$$

where $j^{3}$ denotes the contribution from the bosonic SL(2)-WZW with level $k+2$ and $\psi^{+}, \psi^{-}$are the world-sheet fermions. The compact boson $Y$ corresponds to the degrees of freedom of gauge field which cannot be gauged away. This obeys the twisted boundary condition parameterized by the $\mu$-variable, and the Gaussian factor $e^{-\frac{\pi k}{\tau_{2}}|\mu|^{2}}$ is the contribution of its zero-modes. Note that the range of $\mu$-integral is extended from $\operatorname{Jac}(\Sigma) \cong \Sigma \cong \mathbb{C} / \Lambda,(\Lambda \equiv \mathbb{Z} \tau+\mathbb{Z})$ to the whole complex plane due to the winding modes of $Y$.

- The $z$-parameter ('chemical potential') couples to the left-moving $\mathcal{N}=2 \mathrm{U}(1)$-current given by $[22]$

$$
J=\psi^{+} \psi^{-}+\frac{2}{k}\left(j^{3}+\psi^{+} \psi^{-}\right) \equiv \frac{k+2}{k} \psi^{+} \psi^{-}+\frac{2}{k} j^{3} .
$$

Notice that the Gaussian factor $e^{-\frac{\pi k}{\tau_{2}}|\mu|^{2}}$ includes no dependence on $z$ in the torus partition function (2.2). This should be the case, since $J$ possesses no contribution from the boson $Y$.

Now, the $\mu$-integral in (2.2) is finite as long as $\epsilon>0$, and because of the modular invariance of $\sigma(\tau, \mu, z, \bar{z} ; \epsilon), \boldsymbol{Z}_{\mathrm{reg}}(\tau, z, \bar{z} ; \epsilon)$ is defined so as to preserve the modular property manifestly;

$$
\boldsymbol{Z}_{\mathrm{reg}}(\tau+1, z, \bar{z} ; \epsilon)=\boldsymbol{Z}_{\mathrm{reg}}(\tau, z, \bar{z} ; \epsilon), \quad \boldsymbol{Z}_{\mathrm{reg}}\left(-\frac{1}{\tau}, \frac{z}{\tau}, \frac{\bar{z}}{\bar{\tau}} ; \epsilon\right)=\boldsymbol{Z}_{\mathrm{reg}}(\tau, z, \bar{z} ; \epsilon) .
$$

The partition function $\boldsymbol{Z}_{\mathrm{reg}}(\tau, z, \bar{z} ; \epsilon)$ logarithmically diverges in the limit $\epsilon \rightarrow+0$, and the divergent part is identified with the contributions from the strings propagating in the asymptotic cylindrical region of the cigar. This implies that the characteristic behavior around $\epsilon \rightarrow+0$ is given by

$$
\begin{aligned}
\boldsymbol{Z}_{\mathrm{reg}}(\tau, z, \bar{z} ; \epsilon)= & \mathcal{C}|\ln \epsilon| e^{2 \pi \frac{\hat{c}}{\tau_{2}} z_{1}^{2}}\left|\frac{\theta_{1}(\tau, z)}{\eta(\tau)^{3}}\right|^{2} \\
& \times \sum_{n, w \in \mathbb{Z}} \int_{0}^{\infty} d p q^{\frac{p^{2}}{2}+\frac{1}{4}\left(\frac{n}{\sqrt{k}}+\sqrt{k} w\right)^{2}} \frac{p^{\frac{p^{2}}{2}+\frac{1}{4}\left(\frac{n}{\sqrt{k}}-\sqrt{k} w\right)^{2}}}{y^{\frac{n+k w}{k}}} y^{-\frac{n-k w}{k}} \\
& +\boldsymbol{Z}_{\text {finite }}(\tau, z, \bar{z})+O(\epsilon, \epsilon \ln \epsilon),
\end{aligned}
$$

where $\mathcal{C}$ is some positive constant independent of $\epsilon$. The leading part proportional to $|\ln \epsilon|$ (the 'asymptotic part') is expressible in terms of the extended continuous characters $[18,23]$ as in the free theory when the level $k$ is rational. We here denote the term of order $O\left(\epsilon^{0}\right)$ as 
$\boldsymbol{Z}_{\text {finite }}(\tau, z, \bar{z})$, which is still finite after taking the $\epsilon \rightarrow+0$ limit and is modular invariant. This part can be directly extracted from $\boldsymbol{Z}_{\mathrm{reg}}(\tau, z, \bar{z} ; \epsilon)$ as in [24];

$$
\boldsymbol{Z}_{\text {finite }}(\tau, z, \bar{z})=\lim _{\epsilon \rightarrow+0}\left[1-\epsilon \ln \epsilon \frac{\partial}{\partial \epsilon}\right] \boldsymbol{Z}_{\mathrm{reg}}(\tau, z, \bar{z} ; \epsilon)
$$

We emphasize that $\boldsymbol{Z}_{\text {finite }}(\tau, z, \bar{z})$ is uniquely determined irrespective of the adopted regularization scheme, even though the asymptotic part (the overall constant $\mathcal{C}$ ) as well as the correction terms of $O(\epsilon, \epsilon \ln \epsilon)$ will depend on the method of regularization.

The elliptic genus is obtained by formally setting $\bar{z}=0$ while fixing $z$ at a general complex value in $\boldsymbol{Z}_{\text {finite }}(\tau, z, \bar{z})$. We also have to divide the partition function by a factor

$$
e^{2 \pi \frac{\hat{c}}{\tau_{2}}\left(|z|^{2}-z_{2}^{2}\right)} \equiv e^{2 \pi \frac{\hat{c}}{\tau_{2}}\left(\frac{z+\bar{z}}{2}\right)^{2}} \sim e^{\frac{\pi}{\tau_{2}} \frac{\hat{c}}{2} z^{2}}
$$

in order to include the anomaly factor correctly. In fact, we can uniquely determine this factor by requiring the following conditions due to the analysis given in [6];

- the elliptic genus should have the correct modular property;

$$
\mathcal{Z}(\tau+1, z)=\mathcal{Z}(\tau, z), \quad \mathcal{Z}\left(-\frac{1}{\tau}, \frac{z}{\tau}\right)=e^{i \pi \frac{\hat{c}}{\tau} z^{2}} \mathcal{Z}(\tau, z)
$$

- the elliptic genus should be expanded by the discrete characters (D.2) (with $u=0$ ) around $\tau \sim i \infty$ as

$$
\mathcal{Z}(\tau, z)=\sum \operatorname{ch}_{\mathrm{dis}}(*, 0 ; \tau, z)+[\text { subleading terms }],
$$

with no extra overall factor, where the 'subleading terms' include spectral flow sectors as well as non-holomorphic corrections.

In this way, the elliptic genus is written as

$$
\begin{aligned}
\mathcal{Z}(\tau, z) & =e^{-\frac{\pi}{\tau_{2}} \frac{\hat{c}}{2} z^{2}} \boldsymbol{Z}_{\text {finite }}(\tau, z, \bar{z}=0) \\
& \equiv \lim _{\epsilon \rightarrow+0} e^{-\frac{\pi}{\tau_{2}} \frac{\hat{c}}{2} z^{2}} \boldsymbol{Z}_{\mathrm{reg}}(\tau, z, \bar{z}=0 ; \epsilon) .
\end{aligned}
$$

The equality of second line is due to the simple fact that the asymptotic term in (2.7) drops off when setting $\bar{z}=0$. We thus obtain the path-integral expression of elliptic genus as

$$
\mathcal{Z}(\tau, z)=\lim _{\epsilon \rightarrow+0} k e^{\frac{\pi z^{2}}{k \tau_{2}}} \int_{\mathbb{C}} \frac{d^{2} \mu}{\tau_{2}} \sigma(\tau, \mu, z, 0 ; \epsilon) \frac{\theta_{1}\left(\tau, \mu+\frac{k+2}{k} z\right)}{\theta_{1}\left(\tau, \mu+\frac{2}{k} z\right)} e^{2 \pi i z \frac{\mu_{2}}{\tau_{2}}} e^{-\frac{\pi k}{\tau_{2}}|\mu|^{2}}
$$

The $\mu$-integral is not easy to perform since the Gaussian factor $e^{-\frac{\pi k}{\tau_{2}}|\mu|^{2}}$ breaks the periodicity of the integrand. If this factor was absent, the relevant integral would reduce to a simple period integral over a torus $\Sigma \equiv \mathbb{C} / \Lambda,(\Lambda \equiv \mathbb{Z} \tau+\mathbb{Z})$.

One way to avoid this complication is given by using the following identity [15]

$$
\mathcal{Z}(\tau, z)=\sum_{\lambda \equiv n_{1} \tau+n_{2} \in \Lambda}(-1)^{n_{1}+n_{2}+n_{1} n_{2}} s_{\lambda}^{\left(\frac{\hat{c}}{2}\right)} \cdot \mathcal{Z}^{(\infty)}(\tau, z),
$$


where $s_{\lambda}^{(\kappa)}$ denotes the spectral flow (Eichler-Zagier [25]) operator defined by

$$
\begin{aligned}
s_{\lambda}^{(\kappa)} \cdot f(\tau, z):= & q^{\kappa \alpha^{2}} y^{2 \kappa \alpha} e^{2 \pi i \kappa \alpha \beta} f(\tau, z+\lambda) \\
\equiv & e^{2 \pi i \frac{\kappa}{\tau_{2}} \lambda_{2}(\lambda+2 z)} f(\tau, z+\lambda), \\
& \quad\left(\lambda \equiv \alpha \tau+\beta \equiv \lambda_{1}+i \lambda_{2}, \alpha, \beta, \lambda_{1}, \lambda_{2} \in \mathbb{R}\right) .
\end{aligned}
$$

Recall that the elliptic genus of a complex $D$-dimensional manifold is a Jacobi form with index $\frac{D}{2}$ [25]. Here $\hat{c}(2.1)$ is the effective dimension of a target manifold described by a superconformal field theory with a central charge $c$. Thus the suffix $\frac{\hat{c}}{2}$ of the flow operator $s_{\lambda}^{\left(\frac{\hat{c}}{2}\right)}$ denotes the index of the elliptic genus $\mathcal{Z}(\tau, z)$ describing the cigar geometry.

Since (2.14) is a crucial step in our analysis, we will present its proof in appendix B. What is important here is the fact that the action of the operator $s_{\lambda}^{(\kappa)}$ (as well as the sign factor $(-1)^{n_{1}+n_{2}+n_{1} n_{2}}$ ) preserves the modular covariance, whose precise meaning is given in appendix A.

On the other hand, $\mathcal{Z}^{(\infty)}(\tau, z)$ is defined schematically as the elliptic genus of ' $\mathbb{Z}_{\infty^{-}}$ orbifold' of the cigar model as given in (B.7), or equivalently the universal cover of trumpet in the $T$-dual picture. Namely,

$$
\begin{aligned}
\mathcal{Z}^{(\infty)}(\tau, z) & :=\lim _{\epsilon \rightarrow+0} k e^{\frac{\pi z^{2}}{k \tau_{2}}} \int_{\Sigma} \frac{d^{2} \omega}{\tau_{2}} \int_{\mathbb{C}} \frac{d^{2} \mu}{\tau_{2}} \sigma(\tau, \mu, z, 0 ; \epsilon) \frac{\theta_{1}\left(\tau, \mu+\frac{k+2}{k} z\right)}{\theta_{1}\left(\tau, \mu+\frac{2}{k} z\right)} e^{2 \pi i z \frac{\mu_{2}}{\tau_{2}}} e^{-\frac{\pi k}{\tau_{2}}|\mu+\omega|^{2}} \\
& =\lim _{\epsilon \rightarrow+0} e^{\frac{\pi z^{2}}{k \tau_{2}}} \int_{\Sigma} \frac{d^{2} \mu}{\tau_{2}} \sigma(\tau, \mu, z, 0 ; \epsilon) \frac{\theta_{1}\left(\tau, \mu+\frac{k+2}{k} z\right)}{\theta_{1}\left(\tau, \mu+\frac{2}{k} z\right)} e^{2 \pi i z \frac{\mu_{2}}{\tau_{2}}} .
\end{aligned}
$$

In the second line, due to the periodicity of the integrand under $\mu \rightarrow \mu+\nu, \nu \in \Lambda$ except for the factor $e^{-\frac{\pi k}{\tau_{2}}|\mu+\omega|^{2}}$, we made use of an obvious relation $\int_{\Sigma} \frac{d^{2} \omega}{\tau_{2}} \int_{\mathbb{C}} \frac{d^{2} \mu}{\tau_{2}}[\cdots]=$ $\int_{\mathbb{C}} \frac{d^{2} \omega}{\tau_{2}} \int_{\Sigma} \frac{d^{2} \mu}{\tau_{2}}[\cdots]$, and carried out the Gaussian integral over $\omega$.

Computation is now easy. Since the integrand of (2.16) is holomorphic and periodic with respect to the integration variables $s_{1}, s_{2}$ with $\mu \equiv s_{1} \tau+s_{2}$, one may regard it as a double period integral.

Thus, by deforming the integration contour as

$$
s_{1} \in[0,1]+i \frac{z}{k \tau_{2}}, \quad s_{2} \in[0,1]-i \frac{z \bar{\tau}}{k \tau_{2}},
$$

we can directly evaluate (2.16) with the helps of the identity (A.9) as

$$
\begin{aligned}
\mathcal{Z}^{(\infty)}(\tau, z) & =\lim _{\epsilon \rightarrow+0} e^{-\frac{\pi z^{2}}{k \tau_{2}}} \int_{\Sigma} \frac{d^{2} \mu}{\tau_{2}} \sigma(\tau, \mu, 0,0 ; \epsilon) \frac{\theta_{1}(\tau, \mu+z)}{\theta_{1}(\tau, \mu)} e^{2 \pi i z \frac{\mu_{2}}{\tau_{2}}} \\
& =e^{-\frac{\pi z^{2}}{k \tau_{2}}} \int_{\Sigma} \frac{d^{2} \mu}{\tau_{2}} \frac{\theta_{1}(\tau, \mu+z)}{\theta_{1}(\tau, \mu)} e^{2 \pi i z \frac{\mu_{2}}{\tau_{2}}} \\
& =e^{-\frac{\pi z^{2}}{k \tau_{2}}} \frac{\theta_{1}(\tau, z)}{i \eta(\tau)^{3}} \sum_{n \in \mathbb{Z}} \int_{\Sigma} \frac{d^{2} \mu}{\tau_{2}} \frac{e^{2 \pi i n \mu}}{1-y q^{n}} e^{2 \pi i z \frac{\mu_{2}}{\tau_{2}}} \\
& =e^{-\frac{\pi z^{2}}{k \tau_{2}}} \frac{\theta_{1}(\tau, z)}{2 \pi z \eta(\tau)^{3}} \equiv e^{-\frac{\pi z^{2}}{k \tau_{2}}} \frac{\vartheta(\tau, z)}{z} .
\end{aligned}
$$


In the second line, we have used

$$
\sigma(\tau, \mu, 0,0 ; \epsilon)=\prod_{m_{1}, m_{2} \in \mathbb{Z}}\left[1-e^{-\frac{1}{\epsilon \tau_{2}}\left|\mu+m_{1} \tau+m_{2}\right|^{2}}\right],
$$

as well as the fact that the $\mu$-integral converges even in the absence of $\sigma$-factor. We also introduced the symbol (A.5);

$$
\vartheta(\tau, z) \equiv \frac{\theta_{1}(\tau, z)}{2 \pi \eta(\tau)^{3}} .
$$

Substituting (2.18) back into the 'spectral flow formula' (2.14), we finally obtain the following simple expression of elliptic genus of cigar model;

$$
\begin{aligned}
\mathcal{Z}(\tau, z) & =\sum_{\lambda \equiv n_{1} \tau+n_{2} \in \Lambda}(-1)^{n_{1}+n_{2}+n_{1} n_{2}} s_{\lambda}^{\left(\frac{\hat{c}}{2}\right)} \cdot\left[e^{-\frac{\pi z^{2}}{k \tau_{2}}} \frac{\vartheta(\tau, z)}{z}\right] \\
& =\vartheta(\tau, z) \sum_{\lambda \in \Lambda} s_{\lambda}^{\left(\frac{1}{k}\right)} \cdot\left[\frac{e^{-\frac{\pi z^{2}}{k \tau_{2}}}}{z}\right] \\
& =\vartheta(\tau, z) \sum_{\lambda \in \Lambda} \frac{e^{-\frac{\pi}{k \tau_{2}}\left[z^{2}+|\lambda|^{2}+2 \bar{\lambda} z\right]}}{z+\lambda} \equiv \vartheta(\tau, z) \sum_{\lambda \in \Lambda} \frac{\rho^{(1 / k)}(\lambda, z)}{z+\lambda} .
\end{aligned}
$$

In the last line, we introduced the notation

$$
\rho^{(\kappa)}(\lambda, z):=s_{\lambda}^{(\kappa)} \cdot e^{-\frac{\pi \kappa}{\tau_{2}} z^{2}} \equiv e^{-\frac{\pi \kappa}{\tau_{2}}\left[|\lambda|^{2}+2 \bar{\lambda} z+z^{2}\right]} .
$$

More explicitly, (2.19) is rewritten like a Poincaré series

$$
\mathcal{Z}(\tau, z)=\vartheta(\tau, z) \sum_{m, n \in \mathbb{Z}} q^{\frac{1}{k} m^{2}} y^{\frac{2}{k} m} e^{2 \pi i \frac{m n}{k}} \frac{e^{-\frac{\pi}{k \tau_{2}}(z+m \tau+n)^{2}}}{z+m \tau+n} .
$$

Here the double power series of $\lambda \in \Lambda$ absolutely converges due to the Gaussian factor, and thus (2.19) exhibits good modular behavior. One can easily confirm that the elliptic genus (2.19) possesses the modular property as a weak Jacobi form with weight 0 and index $\hat{c} / 2$ given in (2.10), and also for the case of $k=N / K, N, K \in \mathbb{Z}_{>0}$,

$$
s_{n_{1} \tau+n_{2}}^{\left(\frac{\hat{c}}{2}\right)} \cdot \mathcal{Z}(\tau, z)=(-1)^{n_{1}+n_{2}+n_{1} n_{2}} \mathcal{Z}(\tau, z), \quad\left({ }^{\forall} n_{1}, n_{2} \in N \mathbb{Z}\right) .
$$

\subsection{Relations between the new and old expressions of modular completions}

Now, let us discuss on the relations between present results and our previous analyses given in $[6,15]$, which were based on the 'character decomposition' of the torus partition function.

First of all, we would like to point out that $\mathcal{Z}^{(\infty)}(\tau, z)$ is expanded in terms of modular completions of the irreducible discrete characters,

$$
\mathcal{Z}^{(\infty)}(\tau, z)=\frac{1}{k} \int_{0}^{k} d \nu \widehat{c h}_{\mathrm{dis}}(\nu, 0 ; \tau, z)
$$


as was shown in [15]. Here, the modular completion $\widehat{c h}_{\text {dis }}(\nu, n ; \tau, z)(0 \leq \nu \leq k, n \in \mathbb{Z})$ has been defined by (D.1). It would be helpful to confirm the consistency of the formulas (2.18) and (2.23). In fact, by using (D.1), the r.h.s. of (2.23) is explicitly written as

$$
\frac{1}{k} \int_{0}^{k} d \nu \widehat{c h}_{\mathrm{dis}}(\nu, 0 ; \tau, z)=\frac{1}{k} \frac{\theta_{1}(\tau, z)}{i \eta(\tau)^{3}}\left[\int_{0}^{k} d \nu \frac{y^{\frac{\nu}{k}}}{1-y}+\frac{i}{2 \pi} \int_{-\infty}^{\infty} d \nu \int_{-\infty}^{\infty} d p \frac{y^{\frac{\nu}{k}} e^{-\pi \tau_{2} \frac{p^{2}+\nu^{2}}{k}}}{p-i \nu}\right]
$$

The first term corresponds to the holomorphic part, which just yields

$$
\frac{1}{k} \int_{0}^{k} d \nu \operatorname{ch}_{\operatorname{dis}}(\nu, 0 ; \tau, z) \equiv \frac{1}{k} \frac{\theta_{1}(\tau, z)}{i \eta(\tau)^{3}} \int_{0}^{k} d \nu \frac{y^{\frac{\nu}{k}}}{1-y}=\frac{\vartheta(\tau, z)}{z} .
$$

On the other hand, the double integral in the second term can be evaluated by using the polar coordinate $r e^{i \theta} \equiv p+i \nu$ and elementary properties of Bessel function as follows;

$$
\begin{aligned}
\frac{1}{k} \int_{-\infty}^{\infty} d \nu \int_{-\infty}^{\infty} d p \frac{y^{\frac{\nu}{k}} e^{-\pi \tau_{2} \frac{p^{2}+\nu^{2}}{k}}}{p-i \nu} & =\frac{1}{k} \int_{0}^{\infty} r d r \int_{0}^{2 \pi} d \theta \frac{e^{-\pi \tau_{2} \frac{r^{2}}{k}}}{r e^{-i \theta}} \cdot e^{2 \pi i z \frac{r}{k} \sin \theta} \\
& =-\frac{2 \pi}{k} \int_{0}^{\infty} d r e^{-\pi \tau_{2} \frac{r^{2}}{k}} J_{1}(2 \pi z r / k) \\
& =\sum_{m=0}^{\infty} \frac{(-1)^{m+1}}{(m+1) !}\left(\frac{\pi}{k \tau_{2}}\right)^{m+1} z^{2 m+1} \\
& =\frac{1}{z}\left(e^{-\frac{\pi z^{2}}{k \tau_{2}}}-1\right) .
\end{aligned}
$$

Substituting (2.25) and (2.26) into (2.24), we recover the anticipated formula (2.18);

$$
\begin{aligned}
\mathcal{Z}^{(\infty)}(\tau, z) & =\frac{\vartheta(\tau, z)}{z}+\frac{\vartheta(\tau, z)}{z}\left(e^{-\frac{\pi z^{2}}{k \tau_{2}}}-1\right) \\
& =e^{-\frac{\pi z^{2}}{k \tau_{2}}} \frac{\vartheta(\tau, z)}{z} .
\end{aligned}
$$

We next recall the formula of the elliptic genus of cigar model in the case of $k=N / K$, $\left(N, K \in \mathbb{Z}_{>0}\right)$ given in [6];

$$
\mathcal{Z}(\tau, z)=\frac{\theta_{1}(\tau, z)}{i \eta(\tau)^{3}} \frac{1}{N} \sum_{a, b \in \mathbb{Z}_{N}} s_{a \tau+b}^{(N K)} \cdot \widehat{f}^{(N K)}\left(\tau, \frac{z}{N}\right),
$$

where $\widehat{f}^{(*)}(\tau, z) \equiv \widehat{f}_{0}^{(*)}(\tau, z)$ is the function given in (1.1). Equating this formula with our present result (2.19) in the special case of $N=1, K=1 / k \in \mathbb{Z}_{>0}$, one can rewrite $\widehat{f}^{(K)}$ in a compact form as

$$
\begin{aligned}
\widehat{f}^{(K)}(\tau, z) & =\frac{i}{2 \pi} \sum_{\nu \in \Lambda} \frac{\rho^{(K)}(\nu, z)}{z+\nu} \\
& \equiv \frac{i}{2 \pi} \sum_{m, n \in \mathbb{Z}} q^{K m^{2}} y^{2 K m} \frac{e^{-\frac{\pi K}{\tau_{2}}(z+m \tau+n)^{2}}}{z+m \tau+n} .
\end{aligned}
$$

We shall present a direct proof of this formula in appendix C, with the ' $u$-parameter' included. 
We also recall the relations $[6,15]$

$$
\begin{aligned}
\mathcal{Z}(\tau, z) & =\sum_{\substack{v, a \in \mathbb{Z}_{N} \\
v+K a \in N \mathbb{Z}}} \widehat{\chi}_{\mathrm{dis}}^{(N, K)}(v, a ; \tau, z) \\
& \equiv \sum_{\substack{v, a \in \mathbb{Z}_{N} \\
v+K a \in N \mathbb{Z}}} \sum_{m \in a+N \mathbb{Z}} \widehat{\operatorname{ch}}_{\mathrm{dis}}\left(\frac{v}{K}, m ; \tau, z\right),
\end{aligned}
$$

where $\widehat{\chi}_{\text {dis }}^{(N, K)}$ denotes the modular completion of the discrete extended character (D.4) (for the $u=0$ case). We then likewise obtain

$$
\begin{aligned}
\widehat{\chi}_{\mathrm{dis}}^{(N, K)}(v, a ; \tau, z) & =\vartheta(\tau, z) \sum_{b \in \mathbb{Z}_{N}} \sum_{\lambda \in a \tau+b+N \Lambda} e^{-2 \pi i \frac{b}{N}(v+K a)} \frac{\rho^{(1 / k)}(\lambda, z)}{z+\lambda}, \\
\widehat{\operatorname{ch}}_{\mathrm{dis}}(\nu, m ; \tau, z) & =\vartheta(\tau, z) \sum_{n \in \mathbb{Z}} e^{-2 \pi i \frac{n}{k}(\nu+m)} \frac{\rho^{(1 / k)}(m \tau+n, z)}{z+m \tau+n}
\end{aligned}
$$

We would like to make a comment.

The above manipulations leading to (2.19) is easily generalized to the 'twisted' case;

$$
\mathcal{Z}_{\omega}(\tau, z):=\lim _{\epsilon \rightarrow+0} k e^{\frac{\pi}{k \tau_{2}} z^{2}} \int_{\mathbb{C}} \frac{d^{2} \mu}{\tau_{2}} \sigma(\tau, \mu, z, 0 ; \epsilon) \frac{\theta_{1}\left(\tau, \mu+\frac{k+2}{k} z\right)}{\theta_{1}\left(\tau, \mu+\frac{2}{k} z\right)} e^{2 \pi i z \frac{\mu_{2}}{\tau_{2}}} e^{-\frac{\pi k}{\tau_{2}}|\mu+\omega|^{2}}
$$

where $\omega \in \mathbb{C} / \Lambda(\equiv \Sigma)$ is the twist parameter. (We have an obvious periodicity under $\omega \rightarrow \omega+\nu, \quad{ }^{\prime} \nu \in \Lambda$.)

As is mentioned in appendix B, the formula of 'spectral flow decomposition' (2.14) is generalized to the present case, which is naturally interpreted as the Fourier expansion written as

$$
\mathcal{Z}_{\omega}(\tau, z)=\sum_{\nu \in \Lambda} e^{2 \pi i \frac{1}{\tau_{2}} \operatorname{Im}(\omega \bar{\nu})} \widetilde{\mathcal{Z}}_{\nu}^{(\infty)}(\tau, z)
$$

Here, we introduced

$$
\begin{aligned}
\widetilde{\mathcal{Z}}_{\nu}^{(\infty)}(\tau, z) & :=(-1)^{n_{1}+n_{2}+n_{1} n_{2}} s_{\nu}^{\left(\frac{\hat{c}}{2}\right)} \cdot \mathcal{Z}^{(\infty)}(\tau, z) \\
& \equiv \vartheta(\tau, z) \frac{\rho^{\left(\frac{1}{k}\right)}(\nu, z)}{z+\nu}, \quad\left(\nu \equiv n_{1} \tau+n_{2} \in \Lambda\right),
\end{aligned}
$$

which is also expressed in terms of the modular completion $\widehat{c h}_{\mathrm{dis}}(\lambda, n)$ (D.1) in the same way as $(2.23)$;

$$
\widetilde{\mathcal{Z}}_{\nu}^{(\infty)}(\tau, z)=\frac{1}{k} \int_{0}^{k} d \lambda e^{2 \pi i \frac{n_{2}}{k}\left(\lambda+n_{1}\right)} \widehat{\operatorname{ch}}_{\mathrm{dis}}\left(\lambda, n_{1} ; \tau, z\right) .
$$

The identity (2.34) looks nice. This schematically means that the spectral flow with $\nu \in \Lambda$ is the Fourier transform of the twisting by $\omega \in \mathbb{C} / \Lambda$.

Each manipulation given here keeps the modularity intact. We can readily confirm

$$
\begin{aligned}
\mathcal{Z}_{\omega}(\tau+1, z) & =\mathcal{Z}_{\omega}(\tau, z), & \mathcal{Z}_{\frac{\omega}{\tau}}\left(-\frac{1}{\tau}, \frac{z}{\tau}\right) & =e^{i \pi \frac{\hat{c}}{\tau} z^{2}} \mathcal{Z}_{\omega}(\tau, z), \\
\widetilde{\mathcal{Z}}_{\nu}^{(\infty)}(\tau+1, z) & =\widetilde{\mathcal{Z}}_{\nu}^{(\infty)}(\tau, z), & \widetilde{\mathcal{Z}}_{\frac{\nu}{\tau}}^{(\infty)}\left(-\frac{1}{\tau}, \frac{z}{\tau}\right) & =e^{i \pi \frac{\hat{c}}{\tau} z^{2}} \widetilde{\mathcal{Z}}_{\nu}^{(\infty)}(\tau, z) .
\end{aligned}
$$


One can also evaluate the elliptic genus of the $\mathbb{Z}_{M}$-orbifold of cigar with ${ }^{\forall} M \in \mathbb{Z}_{>0}$ as

$$
\begin{aligned}
\mathcal{Z}^{(M)}(\tau, z) & \equiv \frac{1}{M} \sum_{\gamma \in \Lambda / M \Lambda} \mathcal{Z}_{\frac{\gamma}{M}}(\tau, z) \\
& =\frac{1}{M} \sum_{\nu \in \Lambda} \sum_{\gamma \in \Lambda / M \Lambda} e^{2 \pi i \frac{1}{M \tau_{2}} \operatorname{Im}(\gamma \bar{\nu})} \widetilde{\mathcal{Z}}_{\nu}^{(\infty)}(\tau, z) \quad \because \\
& =M \sum_{\nu \in M \Lambda} \widetilde{\mathcal{Z}}_{\nu}^{(\infty)}(\tau, z) \equiv M \vartheta(\tau, z) \sum_{\nu \in M \Lambda} \frac{\rho^{(1 / k)}(\nu, z)}{z+\nu}
\end{aligned}
$$

More generally, rewriting $\mathcal{Z}_{\gamma}^{(M)}(\tau, z) \equiv \mathcal{Z}_{\gamma / M}(\tau, z)$ as in appendix B, the Fourier transformation is calculated as $\left({ }^{\forall} \lambda \equiv m_{1} \tau+m_{2} \in \Lambda / M \Lambda\right)$;

$$
\begin{aligned}
\widetilde{\mathcal{Z}}_{\lambda}^{(M)}(\tau, z) & \equiv \frac{1}{M} \sum_{\gamma \in \Lambda / M \Lambda} e^{2 \pi i \frac{1}{M \tau_{2}} \operatorname{Im}(\lambda \bar{\gamma})} \mathcal{Z}_{\gamma}^{(M)}(\tau, z) \\
& =M \vartheta(\tau, z) \sum_{\nu \in \lambda+M \Lambda} \frac{\rho^{(1 / k)}(\nu, z)}{z+\nu} \\
& =(-1)^{m_{1}+m_{2}+m_{1} m_{2}} s_{\lambda}^{\left(\frac{\hat{c}}{2}\right)} \cdot \mathcal{Z}^{(M)}(\tau, z) .
\end{aligned}
$$

This result is consistent with the identity (B.5), which is directly checked based on the path-integral expression (2.33) for the special cases $k=N / K, M=N$, as is mentioned in appendix B. It would be worthwhile to note that (2.40) is always correct for ${ }^{\forall} M \in \mathbb{Z}$ and ${ }^{\forall} k \in \mathbb{R}_{>0}$ (not necessarily rational).

\section{$3 \quad$ Extension to general spin structures}

\subsection{Elliptic genus with general spin structures}

In this section, we shall extend the analyses in the previous section to the cases of general spin structure with the continuous parameter of twisting $u \equiv \alpha \tau+\beta,\left({ }^{\forall} \alpha, \beta \in \mathbb{R}\right)$. Namely, we assume the world-sheet fermions of super-gauged WZW model $\psi^{ \pm}(w), \tilde{\psi}^{ \pm}(\bar{w})$ satisfy the twisted boundary condition (with respect to the cylinder coordinate $w, \bar{w}$ );

$$
\begin{array}{rlrl}
\psi^{ \pm}(w+1) & =e^{\mp 2 \pi i \alpha} \psi^{ \pm}(w), & \psi^{ \pm}(w+2 \pi \tau) & =e^{\mp 2 \pi i \beta} \psi(w), \\
\tilde{\psi}^{ \pm}(\bar{w}+1)=e^{ \pm 2 \pi i \alpha} \tilde{\psi}^{ \pm}(\bar{w}), & \tilde{\psi}^{ \pm}(\bar{w}+2 \pi \bar{\tau})=e^{ \pm 2 \pi i \beta} \tilde{\psi}^{ \pm}(\bar{w}) .
\end{array}
$$

In the end, this twist parameter $u$ will turn out to be identified with the ' $u$-variable' of the function $\widehat{f}_{u}^{(*)}(\tau, z)$ introduced in [8]. Closely related studies including such a twisting based on a different approach have been given in $[7,26]$.

We shall again start our analysis from the torus partition function. The partition function for the fermions with boundary condition (3.1) is written concisely by using the spectral flow operator $s_{-u}^{(1 / 2)}$ as

$$
\boldsymbol{Z}_{\text {fermion }}(\tau, z, u)=e^{-\frac{2 \pi}{\tau_{2}} z_{2}^{2}}\left|\frac{s_{-u}^{(1 / 2)} \cdot \theta_{1}(\tau, z)}{\eta(\tau)}\right|^{2},
$$


which is manifestly modular invariant. Thus, the regularized partition function (2.2) is now replaced by

$$
\begin{aligned}
\boldsymbol{Z}_{\mathrm{reg}}(\tau, z, \bar{z}, u ; \epsilon)= & k e^{2 \pi \frac{\hat{c}}{\tau_{2}}|z|^{2}-2 \pi \frac{k+4}{k \tau_{2}} z_{2}^{2}} \int_{\mathbb{C}} \frac{d^{2} \mu}{\tau_{2}} \sigma(\tau, \mu, z, \bar{z} ; \epsilon) \\
& \times\left|\frac{s_{-u}^{(1 / 2)} \cdot \theta_{1}\left(\tau, \mu+\frac{k+2}{k} z\right)}{\theta_{1}\left(\tau, \mu+\frac{2}{k} z\right)}\right|^{2} e^{-4 \pi z_{2} \frac{\mu_{2}}{\tau_{2}}} e^{-\frac{\pi k}{\tau_{2}}|\mu|^{2}} \\
\equiv & k e^{2 \pi \frac{\hat{c}}{\tau_{2}}|z|^{2}-\frac{2 \pi}{k \tau_{2}}\left\{(k+4) X_{2}^{2}+4 X_{2} u_{2}\right\}} \int_{\mathbb{C}} \frac{d^{2} \mu}{\tau_{2}} \sigma(\tau, \mu, X+u, \bar{X}+\bar{u} ; \epsilon) \\
& \times\left|\frac{\theta_{1}\left(\tau, \mu+\frac{k+2}{k} X+\frac{2}{k} u\right)}{\theta_{1}\left(\tau, \mu+\frac{2}{k}(X+u)\right)}\right|^{2} e^{-4 \pi X_{2} \frac{\mu_{2}}{\tau_{2}}} e^{-\frac{\pi k}{\tau_{2}}|\mu|^{2}} .
\end{aligned}
$$

In the second line we set $X:=z-u$ for convenience of calculation given below. Note that we use the notations $z \equiv z_{1}+i z_{2}, u \equiv u_{1}+i u_{2}, X \equiv X_{1}+i X_{2}, \mu \equiv \mu_{1}+i \mu_{2}$, with $z_{i}, u_{i}, X_{i}, \mu_{i} \in \mathbb{R}$, for $i=1,2$.

The elliptic genus is again defined by setting $\bar{z}$ to a particular value while keeping $z$ at a generic complex value. It is easy to find that we have to set $\bar{z}=\bar{u}(\Longleftrightarrow \bar{X}=0)$ to realize the supersymmetric cancellation. We still have to divide by a suitable anomaly factor corresponding to (2.9) in the $u=0$ case. To determine this factor, we require the following conditions which extend (2.10) and (2.11) to the $u \neq 0$ cases:

- the elliptic genus should have the modular property;

$$
\mathcal{Z}(\tau+1, z, u)=\mathcal{Z}(\tau, z, u), \quad \mathcal{Z}\left(-\frac{1}{\tau}, \frac{z}{\tau}, \frac{u}{\tau}\right)=e^{i \pi \frac{\hat{c}}{\tau} z^{2}} \mathcal{Z}(\tau, z, u),
$$

- the elliptic genus should be expanded around $\tau \sim i \infty$ in the form as

$$
\begin{aligned}
\mathcal{Z}(\tau, z, u) & =\sum \operatorname{ch}_{\operatorname{dis}}(*, 0 ; \tau, z, u)+[\text { subleading terms }] \\
& \equiv \sum s_{-u}^{\left(\frac{\hat{c}}{2}\right)} \cdot \operatorname{ch}_{\text {dis }}(*, 0 ; \tau, z)+[\text { subleading terms }] .
\end{aligned}
$$

Then the wanted anomaly factor turns out to be

$$
e^{2 \pi \frac{\hat{c}}{\tau_{2}}\left(z_{1}^{2}+i \frac{u_{2}}{2} \bar{u}\right)} \sim e^{2 \pi \frac{\hat{c}}{\tau_{2}}\left\{\left(\frac{z+\bar{u}}{2}\right)^{2}+i \frac{u_{2}}{2} \bar{u}\right\}} \equiv \rho^{\left(\frac{\hat{c}}{2}\right)}(u, z)^{-1} .
$$

Namely, we define the elliptic genus as

$$
\begin{aligned}
\mathcal{Z}(\tau, z, u):= & \rho^{\left(\frac{\hat{c}}{2}\right)}(u, z) \lim _{\epsilon \rightarrow+0} Z_{\mathrm{reg}}(\tau, z, \bar{z}=\bar{u}, u ; \epsilon) \\
= & e^{-\frac{\pi}{k \tau_{2}}\left(u^{2}-|u|^{2}\right)} e^{i \pi \frac{u_{2}}{\tau_{2}}(u-2 z)} \lim _{\epsilon \rightarrow+0} k e^{\frac{\pi}{k \tau_{2}} X^{2}} \int_{\mathbb{C}} \frac{d^{2} \mu}{\tau_{2}} \sigma(\tau, \mu, X+u, \bar{u} ; \epsilon) \\
& \times \frac{\theta_{1}\left(\tau, \mu+X+\frac{2}{k}(X+u)\right)}{\theta_{1}\left(\tau, \mu+\frac{2}{k}(X+u)\right)} e^{2 \pi i X \frac{\mu_{2}}{\tau_{2}}} e^{-\frac{\pi k}{\tau_{2}}|\mu|^{2}}
\end{aligned}
$$


Perhaps, the simplest way to compute this integral is again to use the 'spectral flow method' that generalizes (2.14);

$$
\mathcal{Z}(\tau, z, u)=\sum_{\lambda \equiv n_{1} \tau+n_{2} \in \Lambda}(-1)^{n_{1}+n_{2}+n_{1} n_{2}} e^{\frac{2 \pi i}{\tau_{2}} \operatorname{Im}(\bar{\lambda} u)} s_{\lambda}^{\left(\frac{\hat{c}}{2}\right)} \cdot \mathcal{Z}^{(\infty)}(\tau, z, u) .
$$

We will give a proof of this identity in appendix B.

$\mathcal{Z}^{(\infty)}(\tau, z, u)$ is evaluated in the same way as (2.16) and (2.18) for the $u=0$ case;

$$
\begin{aligned}
\mathcal{Z}^{(\infty)}(\tau, z, u):= & e^{-\frac{\pi}{k \tau_{2}}\left(u^{2}-|u|^{2}\right)} e^{i \pi \frac{u_{2}}{\tau_{2}}(u-2 z)} \lim _{\epsilon \rightarrow+0} k e^{\frac{\pi X^{2}}{k \tau_{2}}} \int_{\Sigma} \frac{d^{2} \omega}{\tau_{2}} \int_{\mathbb{C}} \frac{d^{2} \mu}{\tau_{2}} \sigma(\tau, \mu, X+u, \bar{u} ; \epsilon) \\
& \times \frac{\theta_{1}\left(\tau, \mu+X+\frac{2}{k}(X+u)\right)}{\theta_{1}\left(\tau, \mu+\frac{2}{k}(X+u)\right)} e^{2 \pi i X \frac{\mu_{2}}{\tau_{2}}} e^{-\frac{\pi k}{\tau_{2}}|\mu+\omega|^{2}} \\
= & e^{-\frac{\pi}{k \tau_{2}}\left(u^{2}-|u|^{2}\right)} e^{i \pi \frac{u_{2}}{\tau_{2}}(u-2 z)} e^{-\frac{\pi}{k \tau_{2}}\left\{X^{2}+2(u-\bar{u}) X\right\}} \int_{\Sigma} \frac{d^{2} \mu}{\tau_{2}} \frac{\theta_{1}(\tau, \mu+X)}{\theta_{1}(\tau, \mu)} e^{2 \pi i X \frac{\mu_{2}}{\tau_{2}}} \\
= & e^{-\frac{\pi}{k \tau_{2}}\left(u^{2}-|u|^{2}\right)} e^{i \pi \frac{u_{2}}{\tau_{2}}(u-2 z)} e^{-\frac{\pi}{k \tau_{2}}\left\{(z-u)^{2}+2(u-\bar{u})(z-u)\right\}} \frac{\vartheta(\tau, z-u)}{z-u} \\
= & \vartheta_{u}(\tau, z) \frac{e^{-\frac{\pi}{k \tau_{2}}\left[z^{2}-2 \bar{u} z+|u|^{2}\right]}}{z-u} \equiv \vartheta_{u}(\tau, z) \frac{\rho^{(1 / k)}(-u, z)}{z-u} .
\end{aligned}
$$

We again made use of the rewriting $\int_{\Sigma} \frac{d^{2} \omega}{\tau_{2}} \int_{\mathbb{C}} \frac{d^{2} \mu}{\tau_{2}}[\cdots]=\int_{\mathbb{C}} \frac{d^{2} \omega}{\tau_{2}} \int_{\Sigma} \frac{d^{2} \mu}{\tau_{2}}[\cdots]$ and carried out the Gaussian integral over $\omega$. In the third line, we again removed the regularization factor $\sigma(* ; \epsilon)$ by a suitable contour deformation, and introduced the symbol

$$
\vartheta_{u}(\tau, z):=s_{-u}^{(1 / 2)} \cdot \vartheta(\tau, z) \equiv e^{i \pi \frac{u_{2}}{\tau_{2}}(u-2 z)} \vartheta(\tau, z-u),
$$

in the last line.

We here note

$$
\mathcal{Z}^{(\infty)}(\tau, z, u)=s_{-u}^{\left(\frac{\hat{c}}{2}\right)} \cdot \mathcal{Z}^{(\infty)}(\tau, z) \equiv s_{-u}^{\left(\frac{\hat{c}}{2}\right)} \cdot\left[\vartheta(\tau, z) \frac{e^{-\frac{\pi z^{2}}{k \tau_{2}}}}{z}\right] .
$$

Therefore, substituting (3.11) into (3.8), we finally obtain

$$
\begin{aligned}
\mathcal{Z}(\tau, z, u) & =\sum_{\lambda \equiv n_{1} \tau+n_{2} \in \Lambda}(-1)^{n_{1}+n_{2}+n_{1} n_{2}} e^{-\frac{2 \pi i}{\tau_{2}} \operatorname{Im}(\lambda \bar{u})} s_{\lambda}^{\left(\frac{\hat{c}}{2}\right)} \cdot s_{-u}^{\left(\frac{\hat{c}}{2}\right)} \cdot\left[\vartheta(\tau, z) \frac{e^{-\frac{\pi z^{2}}{k \tau_{2}}}}{z}\right] \\
& =\vartheta_{u}(\tau, z) \sum_{\lambda \in \Lambda} s_{\lambda}^{\left(\frac{1}{k}\right)} \cdot s_{-u}^{\left(\frac{1}{k}\right)} \cdot\left[\frac{e^{-\frac{\pi z^{2}}{k \tau_{2}}}}{z}\right] \\
& =\vartheta_{u}(\tau, z) \sum_{\lambda \in \Lambda} e^{2 \pi i \frac{1}{k \tau_{2}} \operatorname{Im}(\lambda \bar{u})} s_{\lambda-u}^{\left(\frac{1}{k}\right)} \cdot\left[\frac{e^{-\frac{\pi z^{2}}{k \tau_{2}}}}{z}\right] \\
& =\vartheta_{u}(\tau, z) \sum_{\lambda \in \Lambda-u} \frac{\rho^{(1 / k)}(\lambda, z) e^{2 \pi i \frac{1}{k \tau_{2}} \operatorname{Im}(\lambda \bar{u})}}{z+\lambda} .
\end{aligned}
$$


In the second line we used the identity

$$
s_{n_{1} \tau+n_{2}}^{(1 / 2)} \cdot \vartheta_{u}(\tau, z)=(-1)^{n_{1}+n_{2}+n_{1} n_{2}} e^{2 \pi i \frac{1}{\tau_{2}} \operatorname{Im}(\lambda \bar{u})} \vartheta_{u}(\tau, z),
$$

which is equivalent with (A.14), and we also made use of the product relation of the spectral flow operators (A.11) in the third line.

The main result (3.12) is an extension of the formula (2.19). This obviously shows that $\mathcal{Z}(\tau, z, u)$ possesses the expected modularity (3.4), and also the spectral flow property for the case of $k=N / K, N, K \in \mathbb{Z}_{>0}$ expressed as

$$
s_{n_{1} \tau+n_{2}}^{\left(\frac{\hat{c}}{2}\right)} \cdot \mathcal{Z}(\tau, z, u)=(-1)^{n_{1}+n_{2}+n_{1} n_{2}} \mathcal{Z}(\tau, z, u), \quad\left({ }^{\forall} n_{1}, n_{2} \in N \mathbb{Z}\right) .
$$

One can also write down the extension of the formulas (2.28) and (2.30);

$$
\begin{aligned}
\mathcal{Z}(\tau, z, u) & =\sum_{\substack{v, a \in \mathbb{Z}_{N} \\
v+K a \in N \mathbb{Z}}} \widehat{\chi}_{\text {dis }}^{(N, K)}(v, a ; \tau, z, u) \\
& \equiv-2 \pi i \vartheta_{u}(\tau, z) e^{-\frac{\pi}{\tau_{2}} N K\left(u^{2}-|u|^{2}\right)} \frac{1}{N} \sum_{a, b \in \mathbb{Z}_{N}} s_{a \tau+b}^{(N K)} \cdot \widehat{f}_{u}^{(N K)}\left(\tau, \frac{z}{N}\right),
\end{aligned}
$$

where the modular completion of extended character $\widehat{\chi}_{\mathrm{dis}}^{(N, K)}(v, a ; \tau, z, u)$ is defined in (D.4) and $\widehat{f}_{u}^{(k)}(\tau, z)$ is the function given in (1.1). Comparing (3.12) with (3.14), we obtain a formula, which is the extension of (2.29);

$$
\widehat{f}_{u}^{(k)}(\tau, z)=e^{\frac{\pi k}{\tau_{2}}\left(u^{2}-|u|^{2}\right)} \frac{i}{2 \pi} \sum_{\lambda \in \Lambda-u} \frac{\rho^{(k)}(\lambda, z) e^{2 \pi i \frac{k}{\tau_{2}} \operatorname{Im}(\lambda \bar{u})}}{z+\lambda}, \quad\left(k \in \mathbb{Z}_{>0}\right) .
$$

We present a direct proof of this identity in appendix C.

Based on (3.15), one can readily confirm the following identities for the function $\widehat{f}_{u}^{(k)}(\tau, z) ;^{3}$

$$
\begin{gathered}
\widehat{f}_{u}^{(k)}(\tau+1, z)=\widehat{f}_{u}^{(k)}(\tau, z), \quad \widehat{f}_{\frac{u}{\tau}}^{(k)}\left(-\frac{1}{\tau}, \frac{z}{\tau}\right)=\tau e^{2 \pi i \frac{k}{\tau}\left(z^{2}-u^{2}\right)} \widehat{f}_{u}^{(k)}(\tau, z), \\
\widehat{f}_{u+\omega}^{(k)}(\tau, z+\nu)=e^{-2 \pi i \frac{k}{\tau_{2}}\left[\nu_{2}(\nu+2 z)-\omega_{2}(\omega+2 u)\right]} \widehat{f}_{u}^{(k)}(\tau, z), \quad\left({ }^{\forall} \nu, \omega \in \Lambda\right) .
\end{gathered}
$$

We also note

$$
\widehat{f}_{u+\gamma}^{(k)}(\tau, z+\gamma)=e^{-4 \pi i k \frac{\gamma_{2}}{\tau_{2}}(z-u)} \widehat{f}_{u}^{(k)}(\tau, z), \quad\left({ }^{\forall} \gamma \in \frac{1}{2 k} \mathbb{Z} \tau+\mathbb{R}\right) .
$$

It would be difficult to show the last identity (3.18) directly from the original definition (1.1).

${ }^{3}$ It would be useful to rewrite $(3.15)$ as

$$
\widehat{f}_{u}^{(k)}(\tau, z)=\frac{i}{2 \pi} \sum_{\lambda \in \Lambda} s_{\lambda}^{(k)} \cdot\left[\frac{e^{-\frac{\pi k}{\tau_{2}}\left\{(z-u)^{2}+2(u-\bar{u})(z-u)\right\}}}{z-u}\right],
$$

in order to check the spectral flow relations (3.17) and (3.18). 
Several remarks are in order;

1. Let us assume the rational level $k=N / K, N, K \in \mathbb{Z}_{>0}$. In the similar manner to the analysis given in [6] for the $u=0$ case, one can make the 'character decomposition' of regularized partition function $\boldsymbol{Z}_{\mathrm{reg}}(\tau, z, \bar{z}, u ; \epsilon)$, and extract the modular completions $\widehat{\chi}_{\text {dis }}$ (D.4). Although necessary computations are slightly cumbersome, one can achieve the expected result;

$$
\begin{aligned}
\boldsymbol{Z}_{\mathrm{reg}}(\tau, z, \bar{z}, u ; \epsilon)= & e^{2 \pi \frac{\hat{c}}{\tau_{2}} z_{1}^{2}} \sum_{\begin{array}{c}
v+K\left(a_{L}+a_{R}\right) \in N \mathbb{Z} \\
v, a_{L}, a_{R} \in \mathbb{Z}_{N}
\end{array}} \hat{\chi}_{\mathrm{dis}}^{(N, K)}\left(v, a_{L} ; \tau, z, u\right) \overline{\hat{\chi}_{\mathrm{dis}}^{(N, K)}\left(v, a_{R} ; \tau, z, u\right)} \\
& +[\text { contribution of the continuous characters]. }
\end{aligned}
$$

Note here that (see (D.13))

$$
\left.\overline{\widehat{\chi}_{\text {dis }}^{(N, K)}\left(v, a_{R} ; \tau, z, u\right)}\right|_{\bar{z}=\bar{u}}=\delta_{a_{R}, 0}^{(N)} e^{i \pi \frac{\hat{c}}{\tau_{2}} u_{2} \bar{u}} .
$$

Thus, (3.19) correctly reproduces the elliptic genus (3.14) by setting $\bar{z}=\bar{u}$ and dividing it by the anomaly factor (3.6).

2. The 'twisted elliptic genus' $\mathcal{Z}_{\omega}(\tau, z, u)$ can be also defined by the replacement $e^{-\frac{\pi k}{\tau_{2}}|\mu|^{2}} \longrightarrow e^{-\frac{\pi k}{\tau_{2}}|\mu+\omega|^{2}}$ in the path-integral expression (3.7). We can analyze $\mathcal{Z}_{\omega}(\tau, z, u)$ in a parallel manner as the $u=0$ case and find that

$$
\begin{aligned}
\mathcal{Z}_{\omega}(\tau, z, u) & =\sum_{\nu \in \Lambda} e^{2 \pi i \frac{1}{\tau_{2}} \operatorname{Im}(\omega \bar{\nu})} \widetilde{\mathcal{Z}}_{\nu}^{(\infty)}(\tau, z, u) \\
& \equiv \vartheta_{u}(\tau, z) \sum_{\nu \in \Lambda} e^{2 \pi i \frac{1}{\tau_{2}} \operatorname{Im}\left[\left(\omega-\frac{1}{k} u\right) \bar{\nu}\right]} \frac{\rho^{\left(\frac{1}{k}\right)}(\nu-u, z)}{z-u+\nu}
\end{aligned}
$$

Based on this result, we can also derive the modular transformation formulas

$$
\begin{aligned}
\mathcal{Z}_{\omega}(\tau+1, z, u) & =\mathcal{Z}_{\omega}(\tau, z, u), & \mathcal{Z}_{\frac{\omega}{\tau}}\left(-\frac{1}{\tau}, \frac{z}{\tau}, \frac{u}{\tau}\right) & =e^{i \pi \frac{\hat{c}}{\tau} z^{2}} \mathcal{Z}_{\omega}(\tau, z, u), \\
\widetilde{\mathcal{Z}}_{\nu}^{(\infty)}(\tau+1, z, u) & =\widetilde{\mathcal{Z}}_{\nu}^{(\infty)}(\tau, z, u), & \widetilde{\mathcal{Z}}_{\frac{\nu}{\tau}}^{(\infty)}\left(-\frac{1}{\tau}, \frac{z}{\tau}, \frac{u}{\tau}\right) & =e^{i \pi \frac{\hat{c}}{\tau} z^{2}} \widetilde{\mathcal{Z}}_{\nu}^{(\infty)}(\tau, z, u)
\end{aligned}
$$

3. Recall that the parameter $u$ expresses the general spin structure of world-sheet fermions. However, one should keep it in mind that

$$
s_{-u}^{\left(\frac{\hat{c}}{2}\right)} \cdot \mathcal{Z}(\tau, z) \neq \mathcal{Z}(\tau, z, u)
$$

for generic $u$, despite the relation (3.11). This feature comes from the noncommutativity of the spectral flow operator $s_{\lambda}^{(*)}$. (See (A.11) and also appendix D). Using the twisted elliptic genus $\mathcal{Z}_{\omega}(\tau, z, u)$ given above, we rather obtain

$$
s_{-u}^{\left(\frac{\hat{c}}{2}\right)} \cdot \mathcal{Z}(\tau, z)=\mathcal{Z}_{\frac{2 u}{k}}(\tau, z, u) .
$$


4. As in the $u=0$ case, we can extract the modular completions of extended and irreducible characters (D.4), (D.1) from the elliptic genus (3.12). We thus obtain the useful formulas

$$
\begin{aligned}
\widehat{\chi}_{\mathrm{dis}}^{(N, K)}(v, a ; \tau, z, u)= & \vartheta_{u}(\tau, z) \sum_{b \in \mathbb{Z}_{N}} \sum_{\lambda \in a \tau+b+N \Lambda-u} e^{-2 \pi i\left[\frac{b}{N}(v+K a)-\frac{1}{k \tau_{2}} \operatorname{Im}(\lambda \bar{u})\right]} \\
& \times \frac{\rho^{(1 / k)}(\lambda, z)}{z+\lambda} \\
\widehat{c h}_{\operatorname{dis}}(\nu, m ; \tau, z, u)= & \vartheta_{u}(\tau, z) \sum_{n \in \mathbb{Z}} e^{-2 \pi i\left[\frac{n}{k}(\nu+m)-\frac{1}{k \tau_{2}} \operatorname{Im}\{(m \tau+n) \bar{u}\}\right]} \\
& \times \frac{\rho^{(1 / k)}(m \tau+n-u, z)}{z+m \tau+n-u}
\end{aligned}
$$

which extend (2.31), (2.32).

\subsection{Modular completions of superconformal characters}

As an interesting application of the inclusion of spin structure parameter $u$, let us discuss the modular completion of characters in $\mathcal{N}=2$ and 4 superconformal algebras.

We first look at the modular completions of the $\mathcal{N}=4$ massless characters of general level $k\left(\in \mathbb{Z}_{>0}\right)$.

The character formula of the massless representation of isospin $\ell / 2$, level $k(\ell=$ $0,1, \ldots, k$ ) is given $[9,10,27]$ (in the $\widetilde{\mathrm{R}}$-sector) as

$$
\begin{aligned}
\operatorname{ch}_{0}^{(\widetilde{R})}(k, \ell ; \tau, z) & =(-1)^{k-\ell} \frac{\theta_{1}(\tau, z)^{2}}{i \eta(\tau)^{3} \theta_{1}(\tau, 2 z)} \sum_{a=-\ell}^{\ell+1} \sum_{n \in \mathbb{Z}} \frac{\left(y q^{n}\right)^{a}}{1-y q^{n}} q^{(k+1) n^{2}} y^{2(k+1) n} \\
& \equiv(-1)^{k-\ell} \frac{\theta_{1}(\tau, z)^{2}}{i \eta(\tau)^{3} \theta_{1}(\tau, 2 z)} \sum_{a=-\ell}^{\ell+1} q^{-\frac{a^{2}}{4(k+1)}} s_{\frac{a}{2(k+1)} \tau}^{(k+1)} \cdot f_{\frac{a}{2(k+1)} \tau}^{(k+1)}(\tau, z) .
\end{aligned}
$$

Therefore, it is natural to define its modular completion by replacing $f_{\frac{a}{2(k+1)} \tau}^{(k+1)}(\tau, z)$ with $\widehat{f}_{\frac{a}{2(k+1)}}^{(k+1)} \tau(\tau, z)$;

$$
\widehat{\operatorname{ch}}_{0}^{(\widetilde{R})}(k, \ell ; \tau, z):=(-1)^{k-\ell} \frac{\theta_{1}(\tau, z)^{2}}{i \eta(\tau)^{3} \theta_{1}(\tau, 2 z)} \sum_{a=-\ell}^{\ell+1} q^{-\frac{a^{2}}{4(k+1)}} s_{\frac{a}{2(k+1)}}^{(k+1)} \cdot \widehat{f}_{\frac{a}{2(k+1)} \tau}^{(k+1)}(\tau, z) .
$$

However, because of the identity (3.18), we just obtain

$$
q^{-\frac{a^{2}}{4(k+1)}} s_{\frac{a}{2(k+1)} \tau}^{(k+1)} \cdot \widehat{f}_{\frac{a}{2(k+1)} \tau}^{(k+1)}(\tau, z)=\widehat{f}_{0}^{(k+1)}(\tau, z) .
$$

Hence we find

$$
\widehat{\operatorname{ch}}_{0}^{(\widetilde{\mathrm{R}})}(k, \ell ; \tau, z)=(-1)^{k-\ell} \frac{2(\ell+1) \theta_{1}(\tau, z)^{2}}{i \eta(\tau)^{3} \theta_{1}(\tau, 2 z)} \widehat{f}_{0}^{(k+1)}(\tau, z) \equiv(-1)^{\ell}(\ell+1) \widehat{c h}_{0}^{(\widetilde{R})}(k, 0 ; \tau, z) .
$$

This means that after the modular completion all of the $\mathcal{N}=4$ massless characters for different spins $(\ell)$ collapse to a single function up to an overall constant which is just the 
Witten index. This is a somewhat striking phenomenon but we do not understand its physical significance.

Let's recall the the relation between massless characters and a massive character at the threshold $h=k / 4[9,10,27]$;

$$
\begin{gathered}
\operatorname{ch}_{0}^{(\widetilde{\mathrm{R}})}(k, \ell+1 ; \tau, z)+2 \operatorname{ch}_{0}^{(\widetilde{\mathrm{R}})}(k, \ell ; \tau, z)+\operatorname{ch}_{0}^{(\widetilde{\mathrm{R}})}(k, \ell-1 ; \tau, z)=\operatorname{ch}^{(\widetilde{\mathrm{R}})}\left(k, \ell+1, h=\frac{k}{4} ; \tau, z\right) \\
\equiv-q^{-\frac{(\ell+1)^{2}}{4(k+1)}} \chi_{\ell}^{\mathrm{SU}(2)_{k-1}}(\tau, 2 z) \frac{\theta_{1}(\tau, z)^{2}}{\eta(\tau)^{3}}, \quad(\ell=0,1, \ldots, k-1) .
\end{gathered}
$$

Here, $\chi_{\ell}^{\mathrm{SU}(2)_{k-1}}(\tau, 2 z)$ denotes the spin $\ell / 2$ character of $\mathrm{SU}(2)_{k-1}$;

$$
\chi_{\ell}^{\mathrm{SU}(2)_{k-1}}(\tau, 2 z) \equiv \frac{\Theta_{\ell+1, k+1}(\tau, 2 z)-\Theta_{-\ell-1, k+1}(\tau, 2 z)}{i \theta_{1}(\tau, 2 z)}, \quad(\ell=0,1, \ldots, k-1) .
$$

(3.30) just implies

$$
\widehat{\operatorname{ch}}_{0}^{(\widetilde{\mathrm{R}})}(k, \ell+1 ; \tau, z)+2 \widehat{\mathrm{ch}}_{0}^{(\widetilde{\mathrm{R}})}(k, \ell ; \tau, z)+\widehat{\operatorname{ch}}_{0}^{(\widetilde{\mathrm{R}})}(k, \ell-1 ; \tau, z)=0
$$

Thus one may formally consider that the massive characters at unitarity threshold $\left(h=\frac{k}{4}\right)$ vanish after modular completion;

$$
\widehat{\mathrm{ch}}^{(\widetilde{\mathrm{R}})}\left(k, \ell+1, h=\frac{k}{4} ; \tau, z\right) \equiv 0 .
$$

We can similarly consider the modular completion of the spectrally flowed 'graviton characters' in the $\mathcal{N}=2$ theory of $\hat{c} \equiv 1+\frac{2}{k}$, which is written explicitly as

$$
\begin{aligned}
\operatorname{ch}_{\text {grav }}(n ; \tau, z) & :=-q^{-\frac{1}{4 k}} \frac{(1-q) y q^{n-1}}{\left(1-y q^{n}\right)\left(1-y q^{n-1}\right)} y^{\frac{2}{k}\left(n-\frac{1}{2}\right)} q^{\frac{1}{k}\left(n-\frac{1}{2}\right)^{2}} \frac{\theta_{1}(\tau, z)}{i \eta(\tau)^{3}} \\
& \equiv \operatorname{ch}_{\operatorname{dis}}(-1, n ; \tau, z)-\operatorname{ch}_{\text {dis }}(1, n-1 ; \tau, z)
\end{aligned}
$$

This corresponds to the spectral flow of identity representation with the flow momentum $n \in \mathbb{Z}$ in the NS-sector. Recall the definition of the discrete character (D.2) (for the $u=0$ case);

$$
\operatorname{ch}_{\text {dis }}(\lambda, n ; \tau, z) \equiv \frac{\theta_{1}(\tau, z)}{i \eta(\tau)^{3}} \frac{\left(y q^{n}\right)^{\frac{\lambda}{k}}}{1-y q^{n}} y^{\frac{2 n}{k}} q^{\frac{n^{2}}{k}}
$$

where we allow $\lambda$ to be a general real number.

To make the modular completion of (3.33), one may just replace the second term $\operatorname{ch}_{\text {dis }}(1, n-1)$ with $\widehat{c h}_{\text {dis }}(1, n-1)$. However, the first term $\operatorname{ch}_{\text {dis }}(-1, n ; \tau, z)$ seems subtle, since it does not appear in the expansion of elliptic genus of SL(2)/U(1)-supercoset. Hence, we shall incorporate the dependence on the $u$-variable as is the case of the $\mathcal{N}=4$ characters presented above. Let us note the identity

$$
\operatorname{ch}_{\text {dis }}(-1, n ; \tau, z)=s_{-\frac{k}{2} \tau}^{\left(\frac{\hat{c}}{2}\right)} \cdot \operatorname{ch}_{\text {dis }}\left(k-1, n ; \tau, z, u=-\frac{k}{2} \tau\right)
$$


which is immediately checked from the definition (D.2). Now, we already know how to modular complete the r.h.s. of (3.34). Thus it is natural to define the modular completion of the graviton character (3.33) as

$$
\widehat{c h}_{\text {grav }}(n ; \tau, z):=s_{-\frac{k}{2} \tau}^{\left(\frac{\hat{c}}{2}\right)} \cdot \widehat{c h}_{\text {dis }}\left(k-1, n ; \tau, z,-\frac{k}{2} \tau\right)-\widehat{c h}_{\text {dis }}(1, n-1 ; \tau, z) .
$$

However, we find the identity

$$
s_{\frac{k}{2} j \tau}^{\left(\frac{\hat{c}}{2}\right)} \cdot \widehat{c h}_{\mathrm{dis}}\left(\lambda, n ; \tau, z, \frac{k}{2} j \tau\right)=\widehat{\mathrm{ch}}_{\mathrm{dis}}(\lambda, n ; \tau, z, 0) \equiv \widehat{\operatorname{ch}}_{\mathrm{dis}}(\lambda, n ; \tau, z), \quad\left({ }^{\forall} j \in \mathbb{Z}\right),
$$

which is similar to (3.29). In fact, the formula (3.26) can be rewritten as

$$
\widehat{\operatorname{ch}}_{\text {dis }}(\nu, m ; \tau, z, u)=\vartheta_{u}(\tau, z) \sum_{n \in \mathbb{Z}} e^{-2 \pi i \frac{n}{k}(\nu+m)} s_{m \tau+n}^{\left(\frac{1}{k}\right)} \cdot s_{-u}^{\left(\frac{1}{k}\right)} \cdot\left[\frac{e^{-\frac{\pi z^{2}}{k \tau_{2}}}}{z}\right],
$$

and the commutativity of $s_{\frac{k}{2} j \tau}^{\left(\frac{1}{k}\right)}$ with $s_{m \tau+n}^{\left(\frac{1}{k}\right)}$ readily leads to (3.36). (See (A.11).)

In this way, we have achieved the simple formula of modular completion;

$$
\widehat{c h}_{\text {grav }}(n ; \tau, z)=\widehat{c h}_{\text {dis }}(k-1, n ; \tau, z)-\widehat{c h}_{\text {dis }}(1, n-1 ; \tau, z) .
$$

Because of the periodicity $\widehat{c h}_{\text {dis }}(\lambda, n)=\widehat{c h}_{\text {dis }}(\lambda+k, n)$, one may also rewrite it in the form parallel to (3.33);

$$
\widehat{c h}_{\text {grav }}(n ; \tau, z)=\widehat{c h}_{\text {dis }}(-1, n ; \tau, z)-\widehat{c h}_{\text {dis }}(1, n-1 ; \tau, z) .
$$

Similarly, the extended graviton character $[18,23]$ is written as $\left(a \in \mathbb{Z}_{N}\right)$;

$$
\begin{aligned}
\chi_{\text {grav }}^{(N, K)}(a ; \tau, z) & :=\sum_{n \in a+N \mathbb{Z}} \operatorname{ch}_{\text {grav }}(n ; \tau, z) \\
& \equiv-q^{-\frac{K}{4 N}} \sum_{n \in \mathbb{Z}} \frac{(1-q) y q^{N n+a-1}}{\left(1-y q^{N n+a}\right)\left(1-y q^{N n+a-1}\right)} y^{\frac{2 K}{N}\left(N n+a-\frac{1}{2}\right)} q^{\frac{K}{N}\left(N n+a-\frac{1}{2}\right)^{2}} \frac{\theta_{1}(\tau, z)}{i \eta(\tau)^{3}} . \\
& \equiv \chi_{\text {dis }}^{(N, K)}(-K, a ; \tau, z)-\chi_{\text {dis }}^{(N, K)}(K, a-1 ; \tau, z),
\end{aligned}
$$

and its modular completion should be

$$
\begin{aligned}
\widehat{\chi}_{\text {grav }}^{(N, K)}(a ; \tau, z) & :=\sum_{n \in a+N \mathbb{Z}} \widehat{c h}_{\text {grav }}(n ; \tau, z) \\
& \equiv \widehat{\chi}_{\text {dis }}^{(N, K)}(N-K, a ; \tau, z)-\widehat{\chi}_{\text {dis }}^{(N, K)}(K, a-1 ; \tau, z) \\
& \equiv \widehat{\chi}_{\text {dis }}^{(N, K)}(-K, a ; \tau, z)-\widehat{\chi}_{\text {dis }}^{(N, K)}(K, a-1 ; \tau, z) .
\end{aligned}
$$

In the last line we again made use of the periodicity $\widehat{\chi}_{\mathrm{dis}}^{(N, K)}(v, a)=\widehat{\chi}_{\mathrm{dis}}^{(N, K)}(v+N, a)$. 
It would be worthwhile to note that the 'character identities' in the $\mathcal{N}=2$ case such as (3.31) are written

$$
\begin{aligned}
& \operatorname{ch}_{\text {grav }}(n ; \tau, z)-\operatorname{ch}_{\text {dis }}(k-1, n ; \tau, z)+\operatorname{ch}_{\text {dis }}(1, n-1 ; \tau, z) \\
&= \frac{\theta_{1}(\tau, z)}{i \eta(\tau)^{3}} y^{\frac{1}{k}(2 n-1)} q^{\frac{1}{k} n(n-1)}, \\
& \chi_{\text {grav }}^{(N, K)}(a ; \tau, z)-\chi_{\text {dis }}^{(N, K)}(N-K, a ; \tau, z)+\chi_{\text {dis }}^{(N, K)}(K, a-1 ; \tau, z) \\
&=q^{-\frac{K}{4 N}} \frac{\theta_{1}(\tau, z)}{i \eta(\tau)^{3}} \Theta_{K(2 a-1), N K}\left(\tau, \frac{2 z}{N}\right) .
\end{aligned}
$$

The massive characters appearing in the r.h.s. again vanish after taking the modular completions;

$$
\begin{array}{r}
\widehat{c h}_{\text {grav }}(n ; \tau, z)-\widehat{c h}_{\text {dis }}(k-1, n ; \tau, z)+\widehat{c h}_{\text {dis }}(1, n-1 ; \tau, z)=0, \\
\widehat{\chi}_{\text {grav }}^{(N, K)}(a ; \tau, z)-\widehat{\chi}_{\text {dis }}^{(N, K)}(N-K, a ; \tau, z)+\widehat{\chi}_{\text {dis }}^{(N, K)}(K, a-1 ; \tau, z)=0 .
\end{array}
$$

\section{Acknowledgments}

T.E. would like to thank K.Bringmann for a discussion. Research of T.E. is supported in part by JSPS KAKENHI grant no. 25400273, 22224001 and 23340115.

Y.S. would like to thank S.K. Ashok S. Murthy, B. Pioline, and J. Troost for valuable discussions. He also thanks the organizers of International Workshop on 'Mock Modular Forms and Physics' at IMSc, India, for April 14-18, 2014 for kind hospitality, where part of this work was done. Research of Y.S. is supported in part by JSPS KAKENHI grant no. 23540322 .

\section{A Notations and useful formulas}

In appendix A we summarize the notations adopted in this paper and related useful formulas. We assume $\tau \equiv \tau_{1}+i \tau_{2}, \tau_{2}>0$ and set $q:=e^{2 \pi i \tau}, y:=e^{2 \pi i z}$.

\section{Theta functions.}

$$
\begin{aligned}
\theta_{1}(\tau, z) & =i \sum_{n=-\infty}^{\infty}(-1)^{n} q^{(n-1 / 2)^{2} / 2} y^{n-1 / 2} \equiv 2 \sin (\pi z) q^{1 / 8} \prod_{m=1}^{\infty}\left(1-q^{m}\right)\left(1-y q^{m}\right)\left(1-y^{-1} q^{m}\right), \\
\theta_{2}(\tau, z) & =\sum_{n=-\infty}^{\infty} q^{(n-1 / 2)^{2} / 2} y^{n-1 / 2} \equiv 2 \cos (\pi z) q^{1 / 8} \prod_{m=1}^{\infty}\left(1-q^{m}\right)\left(1+y q^{m}\right)\left(1+y^{-1} q^{m}\right), \\
\theta_{3}(\tau, z) & =\sum_{n=-\infty}^{\infty} q^{n^{2} / 2} y^{n} \equiv \prod_{m=1}^{\infty}\left(1-q^{m}\right)\left(1+y q^{m-1 / 2}\right)\left(1+y^{-1} q^{m-1 / 2}\right), \\
\theta_{4}(\tau, z) & =\sum_{n=-\infty}^{\infty}(-1)^{n} q^{n^{2} / 2} y^{n} \equiv \prod_{m=1}^{\infty}\left(1-q^{m}\right)\left(1-y q^{m-1 / 2}\right)\left(1-y^{-1} q^{m-1 / 2}\right) . \\
\Theta_{m, k}(\tau, z) & =\sum_{n=-\infty}^{\infty} q^{k\left(n+\frac{m}{2 k}\right)^{2}} y^{k\left(n+\frac{m}{2 k}\right)} .
\end{aligned}
$$


We use abbreviations; $\theta_{i}(\tau) \equiv \theta_{i}(\tau, 0)\left(\theta_{1}(\tau) \equiv 0\right), \Theta_{m, k}(\tau) \equiv \Theta_{m, k}(\tau, 0)$. We also set

$$
\eta(\tau)=q^{1 / 24} \prod_{n=1}^{\infty}\left(1-q^{n}\right) .
$$

The spectral flow properties of theta functions are summarized as follows;

$$
\begin{aligned}
\theta_{1}(\tau, z+m \tau+n) & =(-1)^{m+n} q^{-\frac{m^{2}}{2}} y^{-m} \theta_{1}(\tau, z), \\
\theta_{2}(\tau, z+m \tau+n) & =(-1)^{n} q^{-\frac{m^{2}}{2}} y^{-m} \theta_{2}(\tau, z), \\
\theta_{3}(\tau, z+m \tau+n) & =q^{-\frac{m^{2}}{2}} y^{-m} \theta_{3}(\tau, z), \\
\theta_{4}(\tau, z+m \tau+n) & =(-1)^{m} q^{-\frac{m^{2}}{2}} y^{-m} \theta_{4}(\tau, z), \\
\Theta_{a, k}(\tau, 2(z+m \tau+n)) & =e^{2 \pi i n a} q^{-k m^{2}} y^{-2 k m} \Theta_{a+2 k m, k}(\tau, 2 z) .
\end{aligned}
$$

We use the next symbol

$$
\vartheta(\tau, z):=\frac{\theta_{1}(\tau, z)}{\partial_{z} \theta_{1}(\tau, 0)} \equiv \frac{\theta_{1}(\tau, z)}{2 \pi \eta(\tau)^{3}} .
$$

Note that

$$
\lim _{z \rightarrow 0}\left[\partial_{z} \vartheta(\tau, z)\right]=1 .
$$

The modular and spectral flow properties of $\vartheta(\tau, z)$ are summarized as

$$
\begin{aligned}
\vartheta(\tau+1, z) & =\vartheta(\tau, z), \\
\vartheta\left(-\frac{1}{\tau}, \frac{z}{\tau}\right) & =\frac{e^{i \pi \frac{z^{2}}{\tau}}}{\tau} \vartheta(\tau, z), \\
\vartheta(\tau, z+a \tau+b) & =(-1)^{a+b} q^{-\frac{1}{2} a^{2}} y^{-a} \vartheta(\tau, z) .
\end{aligned}
$$

Namely, $\vartheta(\tau, z)$ is a weak Jacobi form of weight -1 and index $\frac{1}{2}$.

The next identity is quite useful for our calculations;

$$
\begin{aligned}
\frac{\theta_{1}(\tau, u+z)}{\theta_{1}(\tau, u)}= & \frac{\theta_{1}(\tau, z)}{i \eta(\tau)^{3}} \sum_{n \in \mathbb{Z}} \frac{w^{n}}{1-y q^{n}}, \\
& \left(y \equiv e^{2 \pi i z}, w \equiv e^{2 \pi i u}, \tau_{2}>0,0<\frac{u_{2}}{\tau_{2}}<1\right) .
\end{aligned}
$$

See e.g. [6] for the proof.

Spectral flow operator. (See also [25].)

$$
\begin{aligned}
& s_{\lambda}^{(\kappa)} \cdot f(\tau, z):=e^{2 \pi i \frac{\kappa}{\tau_{2}} \lambda_{2}(\lambda+2 z)} f(\tau, z+\lambda) \\
& \equiv q^{\kappa \alpha^{2}} y^{2 \kappa \alpha} e^{2 \pi i \kappa \alpha \beta} f(\tau, z+\alpha \tau+\beta), \\
&\left(\lambda \equiv \alpha \tau+\beta,{ }^{\forall} \alpha, \beta \in \mathbb{R}\right) .
\end{aligned}
$$

An important property of the spectral flow operator $s_{\lambda}^{(\kappa)}$ is the modular covariance, which precisely means the following. 
Assume that $f(\tau, z)$ is an arbitrary function with the modular property;

$$
f(\tau+1, z)=f(\tau, z), \quad f\left(-\frac{1}{\tau}, \frac{z}{\tau}\right)=e^{2 \pi i \frac{\kappa}{\tau} z^{2}} \tau^{\alpha} f(\tau, z),
$$

then, we obtain for ${ }^{\forall} \lambda \in \mathbb{C}$

$$
s_{\lambda}^{(\kappa)} \cdot f(\tau+1, z)=s_{\lambda}^{(\kappa)} \cdot f(\tau, z), \quad s_{\frac{\lambda}{\tau}}^{(\kappa)} \cdot f\left(-\frac{1}{\tau}, \frac{z}{\tau}\right)=e^{2 \pi i \frac{\kappa}{\tau} z^{2}} \tau^{\alpha} s_{\lambda}^{(\kappa)} \cdot f(\tau, z) .
$$

The next 'product formula' is also useful;

$$
s_{\lambda}^{(\kappa)} \cdot s_{\lambda^{\prime}}^{(\kappa)}=e^{-2 \pi i \frac{\kappa}{\tau_{2}} \operatorname{Im}\left(\lambda \bar{\lambda}^{\prime}\right)} s_{\lambda+\lambda^{\prime}}^{(\kappa)}=e^{-4 \pi i \frac{\kappa}{\tau_{2}} \operatorname{Im}\left(\lambda \overline{\lambda^{\prime}}\right)} s_{\lambda^{\prime}}^{(\kappa)} \cdot s_{\lambda}^{(\kappa)},
$$

in other words,

$$
s_{\alpha \tau+\beta}^{(\kappa)} \cdot s_{\alpha^{\prime} \tau+\beta^{\prime}}^{(\kappa)}=e^{-2 \pi i \kappa\left(\alpha \beta^{\prime}-\alpha^{\prime} \beta\right)} s_{\left(\alpha+\alpha^{\prime}\right) \tau+\left(\beta+\beta^{\prime}\right)}^{(\kappa)}=e^{-4 \pi i \kappa\left(\alpha \beta^{\prime}-\alpha^{\prime} \beta\right)} s_{\alpha^{\prime} \tau+\beta^{\prime}}^{(\kappa)} \cdot s_{\alpha \tau+\beta}^{(\kappa)} .
$$

We should note that the spectral flow operators do not commute with each other in general.

The next notation has been repeatedly used in the main text;

$$
\vartheta_{u}(\tau, z):=s_{-u}^{(1 / 2)} \cdot \vartheta(\tau, z) \equiv q^{\frac{1}{2} \alpha^{2}} y^{-\alpha} e^{i \pi \alpha \beta} \vartheta(\tau, z-u),
$$

which satisfies

$$
\begin{aligned}
\vartheta_{u}(\tau+1, z) & =\vartheta_{u}(\tau, z), \\
\vartheta_{u / \tau}\left(-\frac{1}{\tau}, \frac{z}{\tau}\right) & =\frac{e^{i \pi \frac{z^{2}}{\tau}}}{\tau} \vartheta_{u}(\tau, z), \\
\vartheta_{u}(\tau, z+m \tau+n) & =(-1)^{m+n} e^{2 \pi i(m \beta-n \alpha)} q^{-\frac{m^{2}}{2}} y^{-m} \vartheta_{u}(\tau, z) .
\end{aligned}
$$

Error function.

$$
\operatorname{Erf}(x):=\frac{2}{\sqrt{\pi}} \int_{0}^{x} e^{-t^{2}} d t, \quad(x \in \mathbb{R}) .
$$

The next identity is elementary but useful;

$$
\operatorname{sgn}(\nu+0)-\operatorname{Erf}(\nu)=\frac{1}{i \pi} \int_{\mathbb{R}-i 0} d p \frac{e^{-\left(p^{2}+\nu^{2}\right)}}{p-i \nu}, \quad(\nu \in \mathbb{R}) .
$$

Weak Jacobi forms. The weak Jacobi form [25] for the full modular group $\Gamma(1) \equiv$ $\mathrm{SL}(2, \mathbb{Z})$ with weight $k\left(\in \mathbb{Z}_{\geq 0}\right)$ and index $r\left(\in \frac{1}{2} \mathbb{Z}_{\geq 0}\right)$ is defined by the conditions

(i) modularity:

$$
\Phi\left(\frac{a \tau+b}{c \tau+d}, \frac{z}{c \tau+d}\right)=e^{2 \pi i r \frac{c z^{2}}{c \tau+d}}(c \tau+d)^{k} \Phi(\tau, z), \quad \forall\left(\begin{array}{ll}
a & b \\
c & d
\end{array}\right) \in \Gamma(1),
$$

(ii) double quasi-periodicity:

$$
\Phi(\tau, z+m \tau+n)=(-1)^{2 r(m+n)} q^{-r m^{2}} y^{-2 r m} \Phi(\tau, z) .
$$


In this paper, we shall use this terminology in a broader sense. We allow a half integral index $r$, and more crucially, allow non-holomorphic dependence on $\tau$, while we keep the holomorphicity with respect to $z .^{4}$

\section{B Proof of (3.8)}

In this appendix we prove the formula (3.8). Even though this identity (for the $u=0$ case) has been already shown in [15] by using the modular completion of irreducible characters, we shall here directly derive it from the path-integral expression of elliptic genus with an arbitrary parameter of continuous spin structure $u$. Of course, we can readily obtain the identity (2.14) by setting $u=0$.

We shall assume $k=N / K$ with some positive integers $N$ and $K$ (not necessarily coprime) for the time being. We will later take the large $N$-limit with keeping $k$ a fixed value. We denote $\Lambda \equiv \mathbb{Z} \tau+\mathbb{Z}$ as in the main text.

Let's start with the orbifolding associated with the $\mathbb{Z}_{N}$-symmetry acting on the angle coordinate $\theta$ of cigar as $\theta \rightarrow \theta+2 \pi \frac{a}{N},\left({ }^{\forall} a \in \mathbb{Z}_{N}\right)$. The corresponding elliptic genus is given as

$$
\mathcal{Z}^{(N)}(\tau, z, u)=\frac{1}{N} \sum_{\gamma \in \Lambda / N \Lambda} \mathcal{Z}_{\gamma}^{(N)}(\tau, z, u)
$$

where each twisted sector is explicitly written as

$$
\begin{aligned}
\mathcal{Z}_{\gamma}^{(N)}(\tau, z, u)= & e^{-\frac{\pi}{k \tau_{2}}\left(u^{2}-|u|^{2}\right)} e^{i \pi \frac{u_{2}}{\tau_{2}}(u-2 z)} \lim _{\epsilon \rightarrow+0} k e^{\frac{\pi}{k \tau_{2}}(z-u)^{2}} \int_{\mathbb{C}} \frac{d^{2} \mu}{\tau_{2}} \sigma(\tau, \mu, z, \bar{u} ; \epsilon) \\
& \times \frac{\theta_{1}\left(\tau, \mu+\frac{k+2}{k} z-u\right)}{\theta_{1}\left(\tau, \mu+\frac{2}{k} z\right)} e^{2 \pi i(z-u) \frac{\mu_{2}}{\tau_{2}}} e^{-\frac{\pi k}{\tau_{2}}\left|\mu+\frac{\gamma}{N}\right|^{2}} .
\end{aligned}
$$

We further introduce their 'Fourier transformation' by the relation as

$$
\widetilde{\mathcal{Z}}_{\delta}^{(N)}(\tau, z, u):=\frac{1}{N} \sum_{\gamma \in \Lambda / N \Lambda} e^{2 \pi i \frac{1}{N \tau_{2}} \operatorname{Im}(\delta \bar{\gamma})} \mathcal{Z}_{\gamma}^{(N)}(\tau, z, u), \quad\left({ }^{\forall} \delta \in \Lambda / N \Lambda\right) .
$$

Now, the next identity is obvious by definition

$$
\mathcal{Z}(\tau, z, u)\left(\equiv \mathcal{Z}_{\gamma=0}^{(N)}(\tau, z, u)\right)=\frac{1}{N} \sum_{\delta \in \Lambda / N \Lambda} \widetilde{\mathcal{Z}}_{\delta}^{(N)}(\tau, z, u)
$$

Moreover, by using the above definitions as well as that of spectral flow operator (A.10), one can directly show the next identity

$$
s_{\lambda}^{\left(\frac{\hat{c}}{2}\right)} \cdot \mathcal{Z}^{(N)}(\tau, z, u)\left(\equiv s_{\lambda}^{\left(\frac{\hat{c}}{2}\right)} \cdot \widetilde{\mathcal{Z}}_{\delta=0}^{(N)}(\tau, z, u)\right)=(-1)^{n_{1}+n_{2}+n_{1} n_{2}} e^{\frac{2 \pi i}{\tau_{2}} \operatorname{Im}(\lambda \bar{u})} \widetilde{\mathcal{Z}}_{\lambda}^{(N)}(\tau, z, u),
$$

for ${ }^{\forall} \lambda \equiv n_{1} \tau+n_{2} \in \Lambda / N \Lambda$.

\footnotetext{
${ }^{4}$ According to the original terminology of [25], the 'weak Jacobi form' of weight $k$ and index $r\left(k, r \in \mathbb{Z}_{\geq 0}\right)$ means that $\Phi(\tau, z)$ should be Fourier expanded as

$$
\Phi(\tau, z)=\sum_{n \in \mathbb{Z} \geq 0} \sum_{\ell \in \mathbb{Z}} c(n, \ell) q^{n} y^{\ell},
$$

in addition to the conditions (A.17) and (A.18). It is called the 'Jacobi form' if it further satisfies the condition: $c(n, \ell)=0$ for ${ }^{\forall} n$, $\ell$ s.t. $4 n r-\ell^{2}<0$.
} 
The identity (B.4) holds for an arbitrarily large $N \in \mathbb{Z}_{>0}$ as long as $k$ is fixed, since $\mathcal{Z}(\tau, z, u)$ depends only on $k$. Therefore, taking the large $N$-limit, and combining the identities (B.4) with (B.5), we eventually obtain

$$
\begin{aligned}
\mathcal{Z}(\tau, z, u) & =\lim _{\substack{N \rightarrow \infty \\
k \equiv N / K: \text { fix }}} \frac{1}{N} \sum_{\lambda \equiv n_{1} \tau+n_{2} \in \Lambda / N \Lambda}(-1)^{n_{1}+n_{2}+n_{1} n_{2}} e^{-\frac{2 \pi i}{\tau_{2}} \operatorname{Im}(\lambda \bar{u})} s_{\lambda}^{\left(\frac{\hat{c}}{2}\right)} \cdot \mathcal{Z}^{(N)}(\tau, z, u) \\
& \equiv \sum_{\lambda \equiv n_{1} \tau+n_{2} \in \Lambda}(-1)^{n_{1}+n_{2}+n_{1} n_{2}} e^{-\frac{2 \pi i}{\tau_{2}} \operatorname{Im}(\lambda \bar{u})} s_{\lambda}^{\left(\frac{\hat{c}}{2}\right)} \cdot \mathcal{Z}^{(\infty)}(\tau, z, u),
\end{aligned}
$$

where we used

$$
\begin{aligned}
\mathcal{Z}^{(\infty)}(\tau, z, u):= & \lim _{\substack{N \rightarrow \infty \\
k \equiv N / K: \text { fix }}} \frac{1}{N} \mathcal{Z}^{(N)}(\tau, z, u) \\
= & e^{-\frac{\pi}{k \tau_{2}}\left(u^{2}-|u|^{2}\right)} e^{i \pi \frac{u_{2}}{\tau_{2}}(u-2 z)} \lim _{\epsilon \rightarrow+0} k e^{\frac{\pi X^{2}}{k \tau_{2}}} \int_{\Sigma} \frac{d^{2} \omega}{\tau_{2}} \int_{\mathbb{C}} \frac{d^{2} \mu}{\tau_{2}} \sigma(\tau, \mu, X+u, \bar{u} ; \epsilon) \\
& \times \frac{\theta_{1}\left(\tau, \mu+\frac{k+2}{k} z\right)}{\theta_{1}\left(\tau, \mu+\frac{2}{k} z\right)} e^{2 \pi i X \frac{\mu_{2}}{\tau_{2}}} e^{-\frac{\pi k}{\tau_{2}}|\mu+\omega|^{2}}
\end{aligned}
$$

in the second line.

In this way, we obtain the desired formula (3.8). Although the above proof was under the assumption $k=N / K,(3.8)$ is correct for ${ }^{\forall} k \in \mathbb{R}$, since we can choose arbitrarily large integers $N$ and $K$. (Recall that $N$ and $K$ are not assumed to be coprime.) (Q.E.D.)

We finally remark the 'Fourier relation' that generalizes (B.6);

$$
\begin{aligned}
& \mathcal{Z}_{\omega}(\tau, z, u):=\lim _{\substack{N \rightarrow \infty \\
k \equiv N / K, \omega \equiv \frac{\gamma}{N}: \text { fix }}} \mathcal{Z}_{\gamma}^{(N)}(\tau, z, u) \\
&= \sum_{\lambda \in \Lambda} e^{\frac{2 \pi i}{\tau_{2}} \operatorname{Im}(\omega \bar{\lambda})} \widetilde{\mathcal{Z}}_{\lambda}^{(\infty)}(\tau, z, u), \\
& \widetilde{\mathcal{Z}}_{\lambda}^{(\infty)}(\tau, z, u):=\lim _{\substack{N \rightarrow \infty \\
k \equiv N / K, \text { fix }}} \frac{1}{N} \widetilde{\mathcal{Z}}_{\lambda}^{(N)}(\tau, z, u) \\
&=(-1)^{n_{1}+n_{2}+n_{1} n_{2}} e^{-2 \pi i \frac{1}{\tau_{2}} \operatorname{Im}(\lambda \bar{u})} s_{\lambda}^{\left(\frac{\hat{c}}{2}\right)} \cdot \mathcal{Z}^{(\infty)}(\tau, z, u), \\
&\left(\lambda \equiv n_{1} \tau+n_{2} \in \Lambda\right) .
\end{aligned}
$$

\section{Proof of (3.15)}

In this appendix we present a proof of the identity (3.15).

We shall first rewrite the original form of $\widehat{f}_{u}^{(k)}(\tau, z)(1.1)$ by an integral formula using the identity (A.16) $(u \equiv \alpha \tau+\beta, \alpha, \beta \in \mathbb{R})$;

$$
\begin{aligned}
\widehat{f}_{u}^{(k)}(\tau, z)= & f_{u}^{(k)}(\tau, z)-\frac{1}{2 \pi i} \sum_{m, r \in \mathbb{Z}} \int_{\mathbb{R}+i(2 k \alpha-0)} d p \frac{e^{-\pi \tau_{2} \frac{p^{2}+(r+2 k \alpha)^{2}}{k}}}{p-i(r+2 k \alpha)}\left(y w^{-1} q^{m}\right)^{r} y^{2 k m} q^{k m^{2}} \\
= & \frac{i}{2 \pi} \sum_{m, r \in \mathbb{Z}}\left\{\int_{\mathbb{R}+i(1-0)} d p-\int_{\mathbb{R}-i 0} d p\left(y w^{-1} q^{m}\right)\right\} \frac{e^{-\pi \tau_{2} \frac{p^{2}+(r+2 k \alpha)^{2}}{k}}}{p-i(r+2 k \alpha)} \\
& \times \frac{\left(y w^{-1} q^{m}\right)^{r}}{1-y w^{-1} q^{m}} y^{2 k m} q^{k m^{2}}
\end{aligned}
$$


Using the identity $\sum_{n \in \mathbb{Z}} e^{2 \pi i n \nu}=\sum_{r \in \mathbb{Z}} \delta(\nu-r)$, we can further rewrite (C.1) as

$$
\begin{aligned}
\widehat{f}_{u}^{(k)}(\tau, z)= & \frac{i}{2 \pi} \sum_{m \in \mathbb{Z}} \int_{-\infty}^{\infty} d \nu \sum_{n \in \mathbb{Z}}\left\{\int_{\mathbb{R}+i} d p-\int_{\mathbb{R}} d p\left(y w^{-1} q^{m}\right)\right\} \frac{e^{-\pi \tau_{2} \frac{p^{2}+(\nu+2 k \alpha)^{2}}{k}}}{p-i(\nu+2 k \alpha)} \\
& \times e^{2 \pi i n \nu} \frac{\left(y w^{-1} q^{m}\right)^{\nu}}{1-y w^{-1} q^{m}} y^{2 k m} q^{k m^{2}} .
\end{aligned}
$$

Here, it is not obvious whether the infinite $n$-summation commutes with the $\nu$-integral as well as the $m$-summation. We thus define

$$
\begin{aligned}
F(\tau, z, u ; \mathcal{N}): & =\frac{i}{2 \pi} \sum_{m \in \mathbb{Z}} \sum_{n=-\mathcal{N}}^{\mathcal{N}} \int_{-\infty}^{\infty} d \nu\left\{\int_{\mathbb{R}+i} d p-\int_{\mathbb{R}} d p\left(y w^{-1} q^{m}\right)\right\} \frac{e^{-\pi \tau_{2} \frac{p^{2}+(\nu+2 k \alpha)^{2}}{k}}}{p-i(\nu+2 k \alpha)} \\
& \times e^{2 \pi i n \nu} \frac{\left(y w^{-1} q^{m}\right)^{\nu}}{1-y w^{-1} q^{m}} y^{2 k m} q^{k m^{2}} \\
\equiv & \sum_{m \in \mathbb{Z}} \sum_{n=-\mathcal{N}}^{\mathcal{N}} s_{m \tau+n}^{(k)} \cdot g^{(k)}(\tau, z, u),
\end{aligned}
$$

where we set

$$
g^{(k)}(\tau, z, u):=\frac{i}{2 \pi} \int_{-\infty}^{\infty} d \nu\left\{\int_{\mathbb{R}+i} d p-\int_{\mathbb{R}} d p\left(y w^{-1}\right)\right\} \frac{e^{-\pi \tau_{2} \frac{p^{2}+(\nu+2 k \alpha)^{2}}{k}}}{p-i(\nu+2 k \alpha)} \frac{\left(y w^{-1}\right)^{\nu}}{1-y w^{-1}} .
$$

The double integral can be explicitly carried out in the same way as (2.26), which yields

$$
\begin{aligned}
g^{(k)}(\tau, z, u) & =\int_{-2 k \alpha}^{1-2 k \alpha} d \nu \frac{\left(y w^{-1}\right)^{\nu}}{1-y w^{-1}}+\frac{i}{2 \pi} \int_{-\infty}^{\infty} d \nu \int_{-\infty}^{\infty} d p \frac{e^{-\pi \tau_{2} \frac{p^{2}+(\nu+2 k \alpha)^{2}}{k}}}{p-i(\nu+2 k \alpha)}\left(y w^{-1}\right)^{\nu} \\
& =\frac{i}{2 \pi} \frac{\left(y w^{-1}\right)^{-2 k \alpha}}{z-u} e^{-\frac{\pi k}{\tau_{2}}(z-u)^{2}} \equiv \frac{i}{2 \pi} \frac{e^{-\frac{\pi k}{\tau_{2}}\left(z^{2}-u^{2}\right)+\frac{2 \pi k}{\tau_{2}} \bar{u}(z-u)}}{z-u}
\end{aligned}
$$

Thus, we obtain

$$
\begin{aligned}
s_{\lambda}^{(k)} \cdot g^{(k)}(\tau, z, u) & \equiv \frac{i}{2 \pi} e^{2 \pi i k \frac{\lambda_{2}}{\tau_{2}}(\lambda+2 z)} \frac{e^{-\frac{\pi k}{\tau_{2}}\left\{(z+\lambda)^{2}-u^{2}\right\}+\frac{2 \pi k}{\tau_{2}} \bar{u}(z+\lambda-u)}}{z+\lambda-u} \\
& =\frac{i}{2 \pi} e^{\frac{\pi k}{\tau_{2}}\left(u^{2}-|u|^{2}\right)} \frac{e^{-\frac{\pi k}{\tau_{2}}\left[z^{2}+2(\bar{\lambda}-\bar{u}) z+|\lambda-u|^{2}-(\lambda \bar{u}-\bar{\lambda} u)\right]}}{z+\lambda-u} \\
& \equiv \frac{i}{2 \pi} e^{\frac{\pi k}{\tau_{2}}\left(u^{2}-|u|^{2}\right)} \frac{\rho^{(k)}(\lambda-u, z) e^{2 \pi i \frac{k}{\tau_{2}} \operatorname{Im}(\lambda \bar{u})}}{z-u+\lambda},
\end{aligned}
$$

for ${ }^{\forall} \lambda \equiv m \tau+n \in \Lambda$.

Thanks to the Gaussian behavior $\sim e^{-\frac{\pi k}{\tau_{2}}|\lambda|^{2}}$ for large $|\lambda|$ in (C.6), we find the absolute convergence;

$$
\sum_{\lambda \in \Lambda}\left|s_{\lambda}^{(k)} \cdot g^{(k)}(\tau, z, u)\right|<\infty
$$


Therefore, we can safely conclude that

$\widehat{f}_{u}^{(k)}(\tau, z)=\lim _{\mathcal{N} \rightarrow \infty} F(\tau, z, u ; \mathcal{N})=\lim _{\mathcal{N} \rightarrow \infty} \sum_{m \in \mathbb{Z}} \sum_{n=-\mathcal{N}}^{\mathcal{N}} s_{m \tau+n}^{(k)} \cdot g^{(k)}(\tau, z, u)=\sum_{\lambda \in \Lambda} s_{\lambda}^{(k)} \cdot g^{(k)}(\tau, z, u)$, which proves the desired identity (3.15). (Q.E.D.)

\section{Summary of modular completions with general spin structures}

In appendix D, we summarize the definitions of modular completions of the irreducible and extended characters of $\mathcal{N}=2$ superconformal algebra with general spin structures parameterized by a continuous parameter $u \in \mathbb{C}$. They are natural extensions of those given in $[6,15]$ for the $u=0$ case. We assume $\tau \in \mathbb{H}$ and set $q \equiv e^{2 \pi i \tau}, y \equiv e^{2 \pi i z}$, $w \equiv e^{2 \pi i u} \equiv e^{2 \pi i(\alpha \tau+\beta)}$.

Modular completions of irreducible characters.

$$
\begin{aligned}
\widehat{\operatorname{ch}}_{\text {dis }}(\lambda, n ; \tau, z, u):= & e^{-\frac{\pi}{k \tau_{2}}\left(u^{2}-|u|^{2}\right)} \vartheta_{u}(\tau, z) \sum_{\nu \in \lambda+2 \alpha+k \mathbb{Z}}\left\{\int_{\mathbb{R}+i(k-0)} d p-\int_{\mathbb{R}-i 0} d p\left(y w^{-1} q^{n}\right)\right\} \\
& \times \frac{e^{-\pi \tau_{2} \frac{p^{2}+\nu^{2}}{k}}}{p-i \nu} \frac{\left(y w^{-1} q^{n}\right)^{\frac{\nu-2 \alpha}{k}}}{1-y w^{-1} q^{n}} y^{\frac{2 n}{k}} q^{\frac{n^{2}}{k}} \\
\equiv & \operatorname{ch}_{\operatorname{dis}}\left([\lambda]_{\alpha}, n ; \tau, z, u\right)-2 \pi i e^{-\frac{\pi}{k \tau_{2}}\left(u^{2}-|u|^{2}\right)} \vartheta_{u}(\tau, z) \\
& \times \sum_{\nu \in \lambda+2 \alpha+k \mathbb{Z}} \int_{\mathbb{R}-i 0} d p \frac{e^{-\pi \tau_{2} \frac{p^{2}+\nu^{2}}{k}}}{p-i \nu}\left(y w^{-1} q^{n}\right)^{\frac{\nu-2 \alpha}{k}} y^{\frac{2 n}{k}} q^{\frac{n^{2}}{k}} \\
\equiv & (-1)^{n} e^{-2 \pi i \beta n} s_{n \tau}^{\left(\frac{\hat{c}}{2}\right)} \cdot s_{-u}^{\left(\frac{\hat{c}}{2}\right)} \cdot \widehat{\operatorname{ch}}_{\mathrm{dis}}(\lambda+2 \alpha, 0 ; \tau, z), \\
& \left(\lambda \in \mathbb{R}, n \in \mathbb{Z},[\lambda]_{\alpha} \equiv \lambda(\bmod k \mathbb{Z}),-2 \alpha \leq[\lambda]_{\alpha} \leq k-2 \alpha\right), \quad \text { (D.1) }
\end{aligned}
$$

and the irreducible character is defined by ${ }^{5}$

$$
\begin{aligned}
\operatorname{ch}_{\text {dis }}(\lambda, n ; \tau, z, u):= & -2 \pi i e^{-\frac{\pi}{k \tau_{2}}\left(u^{2}-|u|^{2}\right)} \vartheta_{u}(\tau, z) \frac{\left(y w^{-1} q^{n}\right)^{\frac{\lambda}{k}}}{1-y w^{-1} q^{n}} y^{\frac{2 n}{k}} q^{\frac{n^{2}}{k}} \\
\equiv & (-1)^{n} e^{-2 \pi i \beta n} s_{n \tau}^{\left(\frac{\hat{c}}{2}\right)} \cdot s_{-u}^{\left(\frac{\hat{c}}{2}\right)} \cdot \operatorname{ch}_{\operatorname{dis}}(\lambda+2 \alpha, 0 ; \tau, z), \\
& (-2 \alpha \leq \lambda \leq k-2 \alpha, n \in \mathbb{Z}) .
\end{aligned}
$$

Here $\operatorname{ch}_{\text {dis }}(\lambda, n ; \tau, z) \equiv \operatorname{ch}_{\text {dis }}(\lambda, n ; \tau, z, u=0)\left(\widehat{\operatorname{ch}}_{\text {dis }}(\lambda, n ; \tau, z) \equiv \operatorname{ch}_{\text {dis }}(\lambda, n ; \tau, z, u=0)\right)$ denotes the (modular completion) of the character associated to the $n$-th spectral flow of discrete irrep. generated by the Ramond vacua;

$$
h=\frac{\hat{c}}{8}, \quad Q=\frac{\lambda}{k}-\frac{1}{2}, \quad(0 \leq \lambda \leq k) .
$$

${ }^{5}$ The factor $e^{-\frac{\pi}{k \tau_{2}}\left(u^{2}-|u|^{2}\right)}$ might look peculiar, since $\operatorname{ch}_{\operatorname{dis}}(\lambda, n ; \tau, z, u)$ should be holomorphic with respect to the modulus $\tau$. However, this is indeed a natural definition from the physical viewpoint, as is implied in the second line of (D.2). Note that $e^{-\frac{\pi}{k \tau_{2}}\left(u^{2}-|u|^{2}\right)} \equiv e^{-\frac{2 \pi i}{k} \alpha(\alpha \tau+\beta)}$, and the 'holomorphicity' here truly means that

$$
\left.\frac{\partial}{\partial \bar{\tau}} \operatorname{ch}_{\operatorname{dis}}(\lambda, n ; \tau, z, \alpha \tau+\beta)\right|_{z, \alpha, \beta: \text { fixed }}=0
$$


Note that the modular completion $\widehat{c h}_{\text {dis }}(\lambda, n)$ has the periodicity under $\lambda \rightarrow \lambda+k$, which is obvious from the definition (D.2), while $\operatorname{ch}_{\text {dis }}(\lambda, n)$ does not.

Modular completions of extended characters. We assume $k=N / K,\left(N, K \in \mathbb{Z}_{>0}\right)$, or equivalently, $\hat{c}=1+\frac{2 K}{N}$.

$$
\begin{aligned}
& \widehat{\chi}_{\text {dis }}^{(N, K)}(v, a ; \tau, z, u):=\sum_{n \in a+N \mathbb{Z}} \widehat{c h}_{\operatorname{dis}}\left(\frac{v}{K}, n ; \tau, z, u\right) \\
& \equiv e^{-\frac{\pi}{k \tau_{2}}\left(u^{2}-|u|^{2}\right)} \vartheta_{u}(\tau, z) \sum_{r \in v+N \mathbb{Z}} \sum_{n \in a+N \mathbb{Z}}\left\{\int_{\mathbb{R}+i(N-0)} d p-\int_{\mathbb{R}-i 0} d p\left(y w^{-1} q^{n}\right)\right\} \\
& \times \frac{e^{-\pi \tau_{2} \frac{p^{2}+(r+2 K \alpha)^{2}}{N K}}}{p-i(r+2 K \alpha)} \frac{\left(y w^{-1} q^{n}\right)^{\frac{r}{N}}}{1-y w^{-1} q^{n}} y^{\frac{2 n}{k}} q^{\frac{n^{2}}{k}} \\
& \equiv \chi_{\text {dis }}^{(N, K)}\left([v]_{\alpha}, a ; \tau, z, u\right)-2 \pi i e^{-\frac{\pi}{k \tau_{2}}\left(u^{2}-|u|^{2}\right)} \vartheta_{u}(\tau, z) \\
& \times \sum_{r \in v+N \mathbb{Z}} \sum_{n \in a+N \mathbb{Z}} \int_{\mathbb{R}-i 0} d p \frac{e^{-\pi \tau_{2} \frac{p^{2}+(r+2 K \alpha)^{2}}{N K}}}{p-i(r+2 K \alpha)}\left(y w^{-1} q^{n}\right)^{\frac{r}{N}} y^{\frac{2 n}{k}} q^{\frac{n^{2}}{k}}, \\
& \left(v, a \in \mathbb{Z}_{N},[v]_{\alpha} \equiv v(\bmod N),-2 K \alpha \leq[v]_{\alpha}<N-2 K \alpha\right),
\end{aligned}
$$

and

$$
\begin{aligned}
\chi_{\mathrm{dis}}^{(N, K)}(v, a ; \tau, z, u):= & \sum_{n \in a+N \mathbb{Z}} \operatorname{ch}_{\operatorname{dis}}\left(\frac{v}{K}, n ; \tau, z, u\right) \\
\equiv & -2 \pi i e^{-\frac{\pi}{k \tau_{2}}\left(u^{2}-|u|^{2}\right)} \vartheta_{u}(\tau, z) \sum_{n \in a+N \mathbb{Z}} \frac{\left(y w^{-1} q^{n}\right)^{\frac{v}{N}}}{1-y w^{-1} q^{n}} y^{\frac{2 n}{k}} q^{\frac{n^{2}}{k}}, \\
& \left(a \in \mathbb{Z}_{N}, v \in \mathbb{Z},-2 K \alpha \leq v \leq N-2 K \alpha\right) .
\end{aligned}
$$

We note that $\chi_{\mathrm{dis}}^{(N, K)}(v, a ; \tau, z) \equiv \chi_{\mathrm{dis}}^{(N, K)}(v, a ; \tau, z, 0)$ is the extended discrete character introduced in $[18,23]$. Again the modular completion $\widehat{\chi}_{\mathrm{dis}}^{(N, K)}(v, a)$ is periodic under $v \rightarrow$ $v+N$, while $\chi_{\text {dis }}^{(N, K)}(v, a)$ is not.

We should remark that, even though

$$
\begin{aligned}
& \widehat{\operatorname{ch}}_{\text {dis }}(\lambda, 0 ; \tau, z, u)=s_{-u}^{\left(\frac{\hat{c}}{2}\right)} \cdot \widehat{\operatorname{ch}}_{\text {dis }}(\lambda+2 \alpha, 0 ; \tau, z), \\
& \operatorname{ch}_{\text {dis }}(\lambda, 0 ; \tau, z, u)=s_{-u}^{\left(\frac{\hat{c}}{2}\right)} \cdot \operatorname{ch}_{\text {dis }}(\lambda+2 \alpha, 0 ; \tau, z),
\end{aligned}
$$

holds by definition, we do not have similar simple relations for $\widehat{c h}_{\text {dis }}(\lambda, n)\left(\operatorname{ch}_{\text {dis }}(\lambda, n)\right)$ with $n \neq 0$ and $\widehat{\chi}_{\mathrm{dis}}^{(N, K)}(v, a)\left(\chi_{\mathrm{dis}}^{(N, K)}(v, a)\right)$. This fact is due to the non-commutativity of the spectral flow operators $s_{\lambda}^{(\kappa)}$, expressed in (A.11). In cases of $K \in 2 \mathbb{Z}_{>0}$, and for $u \in \frac{1}{2} \mathbb{Z} \tau+\frac{1}{2} \mathbb{Z}$, we find

$$
\begin{aligned}
& \widehat{\chi}_{\mathrm{dis}}^{(N, K)}(v, a ; \tau, z, u)=s_{-u}^{\left(\frac{\hat{c}}{2}\right)} \cdot \widehat{\chi}_{\mathrm{dis}}^{(N, K)}(v, a ; \tau, z), \\
& \chi_{\mathrm{dis}}^{(N, K)}(v, a ; \tau, z, u)=s_{-u}^{\left(\frac{\hat{\sigma}}{2}\right)} \cdot \chi_{\mathrm{dis}}^{(N, K)}(v, a ; \tau, z) .
\end{aligned}
$$

Namely, one can define ' $\widehat{\chi}_{\text {dis }}^{(N, K)(\sigma)}(v, a)^{\prime}$ ' (' $\left.\chi_{\text {dis }}^{(N, K)(\sigma)}(v, a)^{\prime}\right)$ with $\sigma=\mathrm{NS}, \widetilde{\mathrm{NS}}, \mathrm{R}$ by the $1 / 2$ spectral flows in the standard manner in those cases. This aspect is consistent with the definitions of modular completions of NS, $\widetilde{\mathrm{NS}}, \mathrm{R}$ sectors given in [24]. 
The modular and spectral flow properties of $\widehat{c h}_{\text {dis }}, \widehat{\chi}_{\text {dis }}$ are almost the same as those given in $[6,15]$;

$$
\begin{aligned}
& \widehat{\operatorname{ch}}_{\text {dis }}(\lambda, n ; \tau+1, z, u)=e^{2 \pi i \frac{n}{k}(\lambda+n)} \widehat{\operatorname{ch}}_{\text {dis }}(\lambda, n ; \tau, z, u), \\
& \widehat{\operatorname{ch}}_{\text {dis }}\left(\lambda, n ;-\frac{1}{\tau}, \frac{z}{\tau}, \frac{u}{\tau}\right)=e^{i \pi \frac{\hat{c}}{\tau} z^{2}} \frac{1}{k} \int_{0}^{k} d \lambda^{\prime} \sum_{n^{\prime} \in \mathbb{Z}} e^{2 \pi i \frac{\lambda \lambda^{\prime}-(\lambda+2 n)\left(\lambda^{\prime}+2 n^{\prime}\right)}{2 k}} \widehat{c h}_{\operatorname{dis}}\left(\lambda^{\prime}, n^{\prime} ; \tau, z, u\right),
\end{aligned}
$$

$$
\begin{aligned}
\widehat{c h}_{\text {dis }}(\lambda, n ; \tau, z+r \tau+s, u) & =(-1)^{r+s} e^{2 \pi i(\beta r-\alpha s)} e^{2 \pi i \frac{\lambda+2 n}{k} s} q^{-\frac{\hat{c}}{2} r^{2}} y^{-\hat{c} r} \widehat{c h}_{\operatorname{dis}}(\lambda, n+r ; \tau, z, u), \\
\widehat{\chi}_{\text {dis }}(v, a ; \tau+1, z, u) & =e^{2 \pi i \frac{a}{N}(v+K a)} \widehat{\chi}_{\text {dis }}(v, a ; \tau, z, u), \\
\widehat{\chi}_{\text {dis }}\left(v, a ;-\frac{1}{\tau}, \frac{z}{\tau}, \frac{u}{\tau}\right) & =e^{i \pi \frac{\hat{c}}{\tau} z^{2}} \frac{1}{N} \sum_{v^{\prime}=0}^{N-1} \sum_{a^{\prime} \in \mathbb{Z}_{N}} e^{2 \pi i \frac{v v^{\prime}-(v+2 K a)\left(v^{\prime}+2 K a^{\prime}\right)}{2 N K}} \widehat{\chi}_{\operatorname{dis}}\left(v^{\prime}, a^{\prime} ; \tau, z, u\right),
\end{aligned}
$$

$$
\widehat{\chi}_{\text {dis }}(v, a ; \tau, z+r \tau+s, u)=(-1)^{r+s} e^{2 \pi i(\beta r-\alpha s)} e^{2 \pi i \frac{v+2 K a}{N} s} q^{-\frac{\hat{c}}{2} r^{2}} y^{-\hat{c} r} \widehat{\chi}_{\operatorname{dis}}(v, a+r ; \tau, z, u),
$$

$$
\left({ }^{\forall} r, s \in \mathbb{Z}\right) \text {. }
$$

We also note the formula for Witten indices;

$$
\begin{aligned}
\lim _{z \rightarrow u} \operatorname{ch}_{\mathrm{dis}}(\lambda, n ; \tau, z, u) & =\lim _{z \rightarrow u} \widehat{c h}_{\mathrm{dis}}(\lambda, n ; \tau, z, u)=\delta_{n, 0} e^{-i \pi \hat{c} \alpha u}, \\
\lim _{z \rightarrow u} \chi_{\mathrm{dis}}^{(N, K)}(v, a ; \tau, z, u) & =\lim _{z \rightarrow u} \widehat{\chi}_{\mathrm{dis}}^{(N, K)}(v, a ; \tau, z, u)=\delta_{a, 0}^{(N)} e^{-i \pi \hat{c} \alpha u} \\
& \equiv \begin{cases}e^{-i \pi \hat{c} \alpha u} & a \equiv 0(\bmod N) \\
0 & \text { otherwise. }\end{cases}
\end{aligned}
$$

Open Access. This article is distributed under the terms of the Creative Commons Attribution License (CC-BY 4.0), which permits any use, distribution and reproduction in any medium, provided the original author(s) and source are credited.

\section{References}

[1] E. Witten, On string theory and black holes, Phys. Rev. D 44 (1991) 314 [INSPIRE].

[2] G. Mandal, A.M. Sengupta and S.R. Wadia, Classical solutions of two-dimensional string theory, Mod. Phys. Lett. A 6 (1991) 1685 [InSPIRE].

[3] I. Bars and D. Nemeschansky, String Propagation in Backgrounds With Curved Space-time, Nucl. Phys. B 348 (1991) 89 [InSPIRE].

[4] S. Elitzur, A. Forge and E. Rabinovici, Some global aspects of string compactifications, Nucl. Phys. B 359 (1991) 581 [inSPIRE].

[5] J. Troost, The non-compact elliptic genus: mock or modular, JHEP 06 (2010) 104 [arXiv: 1004.3649] [INSPIRE].

[6] T. Eguchi and Y. Sugawara, Non-holomorphic Modular Forms and $\mathrm{SL}(2, \mathbb{R}) / \mathrm{U}(1)$ Superconformal Field Theory, JHEP 03 (2011) 107 [arXiv: 1012.5721] [INSPIRE].

[7] S.K. Ashok and J. Troost, A twisted non-compact elliptic genus, JHEP 03 (2011) 067 [arXiv: 1101.1059] [INSPIRE]. 
[8] S. Zwegers, Mock Theta functions, Ph.D. Thesis, Utrecht University, Utrecht, Netherlands (2002), arXiv:0807.4834 [INSPIRE].

[9] T. Eguchi and A. Taormina, Character Formulas for the $N=4$ Superconformal Algebra, Phys. Lett. B 200 (1988) 315 [INSPIRE].

[10] T. Eguchi and A. Taormina, On the Unitary Representations of $N=2$ and $N=4$ Superconformal Algebras, Phys. Lett. B 210 (1988) 125 [INSPIRE].

[11] S.K. Ashok and J. Troost, Elliptic genera and real Jacobi forms, JHEP 01 (2014) 082 [arXiv: 1310.2124] [INSPIRE].

[12] S.K. Ashok, N. Doroud and J. Troost, Localization and real Jacobi forms, JHEP 04 (2014) 119 [arXiv:1311.1110] [INSPIRE].

[13] S. Murthy, A holomorphic anomaly in the elliptic genus, JHEP 06 (2014) 165 [arXiv: 1311.0918] [INSPIRE].

[14] K. Hori and A. Kapustin, World sheet descriptions of wrapped NS five-branes, JHEP 11 (2002) 038 [hep-th/0203147] [INSPIRE].

[15] Y. Sugawara, Comments on Non-holomorphic Modular Forms and Non-compact Superconformal Field Theories, JHEP 01 (2012) 098 [arXiv:1109.3365] [INSPIRE].

[16] A. Hanany, N. Prezas and J. Troost, The partition function of the two-dimensional black hole conformal field theory, JHEP 04 (2002) 014 [hep-th/0202129] [INSPIRE].

[17] D. Israel, A. Pakman and J. Troost, Extended $\mathrm{SL}(2, \mathbb{R}) / \mathrm{U}(1)$ characters, or modular properties of a simple nonrational conformal field theory, JHEP 04 (2004) 043 [hep-th/0402085] [INSPIRE].

[18] T. Eguchi and Y. Sugawara, $\mathrm{SL}(2, \mathbb{R}) / \mathrm{U}(1)$ supercoset and elliptic genera of noncompact Calabi-Yau manifolds, JHEP 05 (2004) 014 [hep-th/0403193] [INSPIRE].

[19] K. Gawędzki and A. Kupiainen, Coset Construction from Functional Integrals, Nucl. Phys. B 320 (1989) 625 [inSPIRE].

[20] H.J. Schnitzer, A Path Integral Construction of Superconformal Field Theories From a Gauged Supersymmetric Wess-Zumino-Witten Action, Nucl. Phys. B 324 (1989) 412 [INSPIRE].

[21] D. Karabali and H.J. Schnitzer, BRST Quantization of the Gauged WZW Action and Coset Conformal Field Theories, Nucl. Phys. B 329 (1990) 649 [InSPIRE].

[22] Y. Kazama and H. Suzuki, New $N=2$ Superconformal Field Theories and Superstring Compactification, Nucl. Phys. B 321 (1989) 232 [INSPIRE].

[23] T. Eguchi and Y. Sugawara, Modular bootstrap for boundary $N=2$ Liouville theory, JHEP 01 (2004) 025 [hep-th/0311141] [INSPIRE].

[24] Y. Sugawara, Thermodynamics of Superstring on Near-extremal NS5 and Effective Hagedorn Behavior, JHEP 10 (2012) 159 [arXiv:1208.3534] [INSPIRE].

[25] M. Eichler and D. Zagier, The Theory of Jacobi Forms, Progress of Mathematics 55 (1985), Springer.

[26] S.K. Ashok, E. Dell'Aquila and J. Troost, Higher Poles and Crossing Phenomena from Twisted Genera, JHEP 08 (2014) 087 [arXiv: 1404.7396] [INSPIRE].

[27] T. Eguchi, Y. Sugawara and A. Taormina, Modular Forms and Elliptic Genera for ALE Spaces, arXiv: 0803.0377 [INSPIRE]. 